\begin{tabular}{|c|c|}
\hline Title & A erodynamic Heating Prediction of Flare type Membrane Inflatable Reentry V ehicle from Low Earth Orbit \\
\hline Author(s) & Koike, Taiki; T akahashi, Y usuke; O shima, Nobuyuki; Y amada, Kazuhiko \\
\hline Citation & $\begin{array}{l}2018 \text { A IA A A tmospheric Flight Mechanics Conference } \\
\text { https://doi.org/10.2514/6.2018-0289 }\end{array}$ \\
\hline Issue Date & $2018-01$ \\
\hline Doc URL & http:/hdl.handle.net/2115/71351 \\
\hline Type & proceedings (author version) \\
\hline File Information & A erodynamic Heating Prediction of Flare type Membrane Inflatable Reentry V ehicle from Low Earth Orbit.pdf \\
\hline
\end{tabular}

Instructions for use 


\title{
Aerodynamic Heating Prediction of Flare-type Membrane Inflatable Reentry Vehicle from Low Earth Orbit
}

\author{
Taiki Koike*, Yusuke Takahashi ${ }^{\dagger}$ and Nobuyuki Oshima* \\ Hokkaido University, \\ Kita 13 Nishi 8, Kita-ku, Sapporo, Hokkaido, 060-8628, Japan \\ and \\ Kazuhiko Yamada ${ }^{\S}$ \\ Japan Aerospace Exploration Agency, \\ 3-1-1 Yoshinodai Chuo-ku, Sagamihara, Kanagawa, 252-5210, Japan
}

Aerodynamic heating around a flare-type membrane inflatable vehicle during Earth atmospheric reentry was investigated using numerical simulation approach. This vehicle, which is mainly composed of the capsule, membrane aeroshell and inflatable torus, has been developed by the Membrane Aeroshell for Atmosphericentry Capsule (MAAC) group as a one of the innovative reentry systems. Analysis solver for reentry flows around the vehicle was RG-FaSTAR, which is a branch version of JAXA fast aerodynamic routine (FaSTAR). In addition, structure analysis solver also was used for membrane deformation in a loosely-coupled manner with the flow field. In the present research, the effects of angle of attack (AoA) and membrane aeroshell deformation on aerodynamic heating were investigated. The numerical results showed that heat flux distribution drastically varies with the increase in AoA because of changes of flow field, and heat flux value at the stagnation point for case of AoA of 40 degree was 3.09 times as high as that for 0 degree. Moreover, the deformed shapes for case of $\mathrm{AoA}$ of 0 and 40 degrees were calculated in the way which the pressure distributions obtained using initial (undeformed) shape were given as the aerodynamic force. The difference of heat fluxes between the deformed and initial shapes on the head capsule part was remarkable as $188.8 \%$ for case of AoA of 0 degree. On the other hand, it was indicated that membrane deformation for case of AoA of 40 degree insignificantly affects the peak heat flux value on the inflatable torus such as the case of the AoA of 0 degree.

\section{Nomenclature}

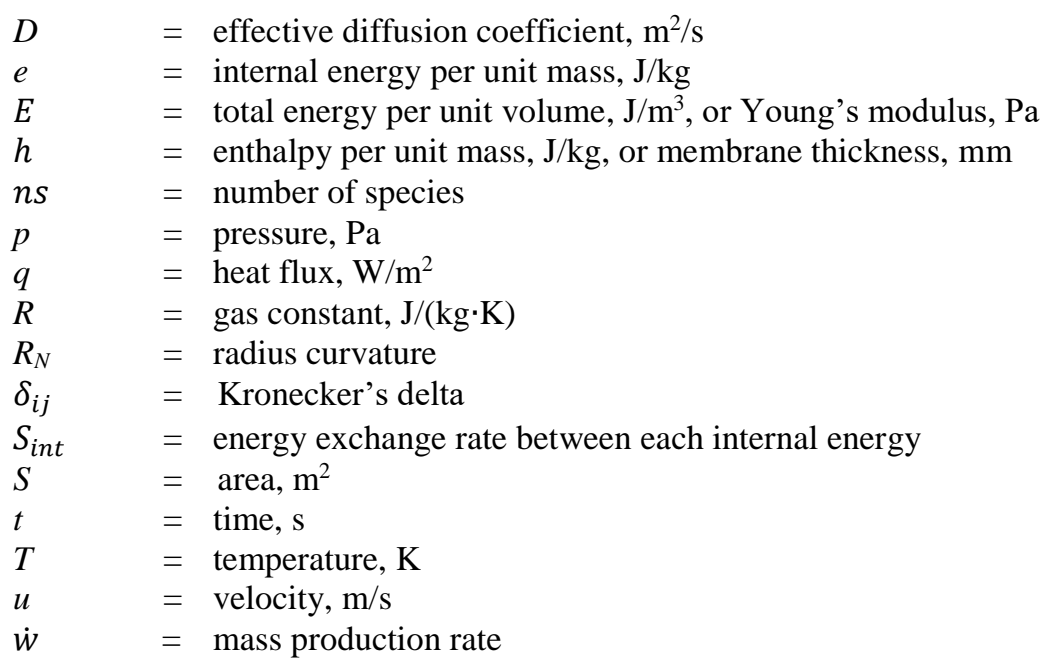

\footnotetext{
* Graduation student, Graduate School of Engineering.

$\dagger$ Assistant Professor, Faculty of Engineering.

* Professor, Faculty of Engineering.

$\S$ Associate Professor, Institute of Space and Astronautical Science.
}

American Institute of Aeronautics and Astronautics 


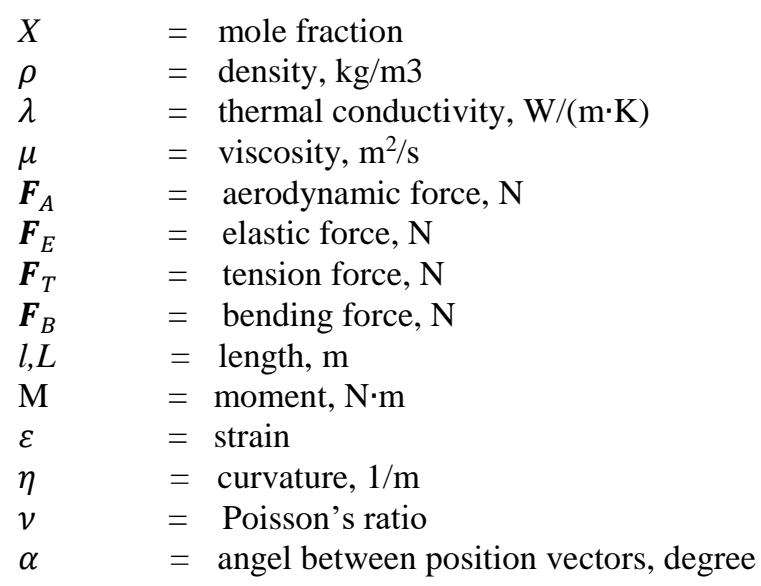

\section{Subscripts}

$S \quad=$ species

$\infty \quad=$ free stream

\section{Introduction}

A reentry vehicle with a membrane aeroshell deployable by an inflatable torus structure (inflatable reentry vehicle) has been developed as a candidate for a next generation space transport system. The main feature of note for this kind of vehicle, which was originally proposed in the 1960s, is reduction in aerodynamic heating because of low ballistic coefficient during atmospheric reentry. This directly contributes to a decrease in the heat flux on the surface of this vehicle. So far, several studies and demonstration flights regarding to this kind of vehicle have been performed by the National Aeronautics and Space Administration (NASA), the European Space Technology (ESA), and the Japan Aerospace Exploration Agency (JAXA).

Inflatable reentry vehicle is composed by capsule, flare-type membrane aeroshell, and inflatable torus. In the considered reentry mission, a capsule with a compactly packed aeroshell is transported to a given orbit as a first step. And then as the inflatable torus tube is inflated, the vehicle rapidly expands the membrane aeroshell under vacuum and microgravity conditions. This membrane is sustained by the inflatable torus. This vehicle re-enters with low ballistic coefficient during atmospheric reentry because of the large area and light weight of the aeroshell. In addition, because the aeroshell deployment is finished before deorbit, a critical operation such as parachute extraction is possibly dispensed in exchange for the use of the inflatable vehicle during the EDL approach.

For research of flare-type thin-membrane aeroshell, several studies of elemental technologies and demonstration flight have been performed as part of the Membrane Aeroshell for Atmospheric-entry Capsule (MAAC) project by JAXA and several universities. Free flight tests using a scientific balloon were performed in 2004 and $2009^{1,2}$. The aerodynamic heating in front of an inflatable vehicle in JAXA's hypersonic wind tunnel was evaluated using a numerical simulation method ${ }^{3}$. The structural strength of a membrane aeroshell was examined using JAXA's lowspeed wind tunnel ${ }^{4}$. Because the plasma behind the shock wave becomes weak due to the low ballistic coefficient flight, the possibility of vehicle-to-ground-station communication increases. In Ref ${ }^{5,6}$, the behavior of the electromagnetic waves around the vehicle during atmospheric reentry was numerically investigated, and the reduction of radio frequency blackout was indicated.

Important milestones in the MAAC project include two reentry demonstrations. One is a reentry demonstration using a sounding rocket ${ }^{7-11}$ (SMAAC: Sounding Rocket Experiment of MAAC) to clarify low ballistic coefficient flight of the inflatable vehicle during reentry. The other is reentry from an altitude of 400km (EGG: Re-Entry Satellite with Gossamer Aeroshell and GPS/Iridium) to demonstrate deployment of the flare-type membrane aeroshell on orbit, communication with the ground using GPS/Iridium network, and orbital decay by aerodynamic deceleration due to the inflatable structure ${ }^{12}$.

One of results of SMAAC suggested that this vehicle re-entered with angle of attack (AoA) and it is necessary to investigate the influence of angle of attack (AoA).

American Institute of Aeronautics and Astronautics 
Aerodynamic heating for inflatable reentry vehicle with AoA was measured using JAXA's hypersonic wind tunnel, and then, a numerical simulation model was developed by Matsunaga et $\mathrm{al}^{13}$. As a result, the numerical simulation approach reproduced aerodynamic heating in hypersonic region within 10\% errors compared with the experiment. Takahashi et al ${ }^{14}$ conducted numerical analysis for SMAAC to investigate the effect of membrane aeroshell deformation because SMAAC surface is largely deformed by aerodynamic force due to introduction of flexible material at the membrane aeroshell ${ }^{3}$. It is elucidated by this work that aerodynamic behaviors on the surface with deformation changed completely comparing undeformed membrane aeroshell.

As one of the final goal of MAAC project, a reentry demonstration mission from the low Earth orbit has been planned (TITANS). To achieve this test flight, aerodynamic heating of the vehicle during atmospheric reentry need to be clarified. However, it is hard to sufficiently reproduce the environment which a reentry vehicle is heated due to aerodynamic heating by using ground facilities, such as hypersonic wind tunnel, arc-heated wind tunnel, and ICP heater. In the last few decades, remarkable progress has been achieved in the high-performance computers and algorithms for numerical simulation. Computational fluid dynamics (CFD) technique has been an effective tool for prediction of various flow field. In the present paper, we investigate the aerodynamic heating environment around TITANS at hypersonic speeds by CFD, focusing on the influences of AoA and membrane aeroshell deformation.

\section{Formulations}

\section{A. Analysis Object}

Shape and analysis model of TITANS are shown in Figs. 1 and 2, respectively. The diameter is $2.5 \mathrm{~m}$ and the structure of TITANS is sustained by the inflatable torus pressuring gas. Analysis model was made based on CAD data.

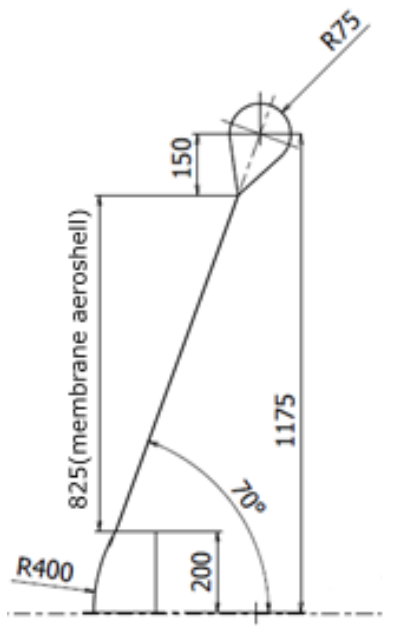

Figure 1. Configuration of TITANS (all dimensions are in milimeters)

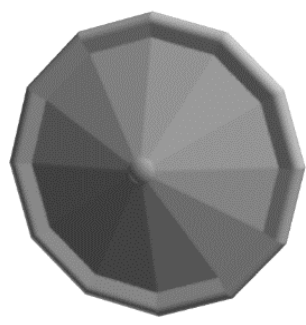

Front side view

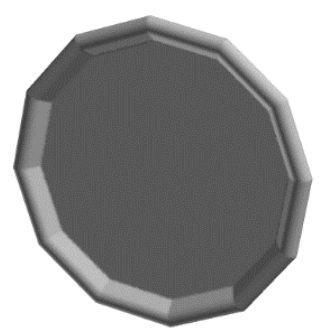

Rear side view

Figure 2. Analysis model of TITANS 


\section{B. Flow Field Model}

The following assumptions are employed here: (1) The flow is steady state, continuum and laminar; (2) The gas considered is real gas; (3) The flow is in thermo chemical non-equilibrium.

The flow field is described by solving the compressible Navier-Stakes equations. The governing equations are composed of the total mass, momentum, total energy conservations. In addition, mass conservation for each chemical species, rotation, vibration and electronic energy conservations are applied in thermo chemical non-equilibrium flow. All equations can be expressed as follows:

$$
\begin{gathered}
\frac{\partial \rho}{\partial t}+\frac{\partial\left(\rho u_{j}\right)}{\partial x_{j}}=0 \\
\frac{\partial\left(\rho u_{j}\right)}{\partial t}+\frac{\partial\left(\rho u_{i} u_{j}+\delta_{i j} p\right)}{\partial x_{j}}=\frac{\partial}{\partial x_{j}}\left[\mu\left(\frac{\partial u_{i}}{\partial x_{j}}+\frac{\partial u_{j}}{\partial x_{i}}\right)-\frac{2}{3} \mu \frac{\partial u_{k}}{\partial x_{k}} \delta_{i j}\right] \\
\frac{\partial E}{\partial t}+\frac{\partial}{\partial x_{j}}\left[(E+p) u_{j}\right] \\
=\frac{\partial}{\partial x_{j}}\left(\lambda_{t r} \frac{\partial T_{t r}}{\partial x_{j}}+\lambda_{r o t} \frac{\partial T_{r o t}}{\partial x_{j}}+\lambda_{v i b} \frac{\partial T_{v i b}}{\partial x_{j}}+\lambda_{e} \frac{\partial T_{e}}{\partial x_{j}}\right) \\
+\frac{\partial}{\partial x_{j}}\left(\rho \sum_{s=1}^{n s} h_{s} D_{s} \frac{\partial X_{s}}{\partial x_{j}}\right) \\
+\frac{\partial}{\partial x_{j}}\left[u_{j} \mu\left(\frac{\partial u_{i}}{\partial x_{j}}+\frac{\partial u_{j}}{\partial x_{i}}\right)-\frac{2}{3} u_{j} \mu \frac{\partial u_{k}}{\partial x_{k}} \delta_{i j}\right] \\
\frac{\partial E_{v i b}}{\partial t}+\frac{\partial\left(E_{v i b} u_{j}\right)}{\partial x_{j}}=\frac{\partial}{\partial x_{j}}\left(\lambda_{v i b} \frac{\partial T_{v i b}}{\partial x_{j}}\right)+\frac{\partial}{\partial x_{j}}\left(\rho \sum_{s=M} e_{v i b, s} D_{s} \frac{\partial X_{s}}{\partial x_{j}}\right)+S_{\text {int }, v i b} \\
\frac{\partial\left(E_{e} u_{j}\right)}{\partial x_{j}}=\frac{\partial}{\partial x_{j}}\left(E_{j} u_{j}\right) \\
\left.\left.\frac{\partial x_{j}}{\partial x_{j}}=\frac{\partial T_{e}}{\partial x_{j}}\right)+\frac{\partial}{\partial x_{j}}\left(\rho \sum_{s=M} e_{e, s} D_{s} \frac{\partial X_{s}}{\partial x_{j}}\right)+S_{\text {int }, e} \frac{\partial X_{r o t}}{\partial x_{j}}\right)+\frac{\partial}{\partial x_{j}}\left(\rho \sum_{s=M} e_{r o t, s} D_{s} \frac{\partial X_{s}}{\partial x_{j}}\right)+S_{\text {int }, r o t} \\
\lambda_{j}
\end{gathered}
$$

where $\delta_{i j}, E, \lambda, h, D, X, \dot{w}, e, S_{i n t}$ and $p$ are the Kronecker's delta, internal energy per unit volume, heat transfer coefficient, enthalpy per unit mass, effective diffusion coefficient, mole fraction, mass production rate, internal energy per unit mass, energy exchange rate between each internal energy and pressure. Subscripts of tr, rot and vib are translation, rotation and vibration respectively. Chapman-Enskog theory is applied to calculate transport properties of mixture gas. Gupta's model ${ }^{15}$ and Fertig's model ${ }^{16}$ are adopted to calculate collision cross section. 49 chemical reactions for 11 chemical species are considered in high temperature air ${ }^{17-19}$. 


\section{Software Package: RG-FaSTAR}

In the present study, RG-FaSTAR v2.0.0 $0^{20}$ is adopted. RG-FaSTAR is a high-enthalpy flow solver that incorporates real gas effects and thermos chemical non-equilibrium, and is a version of the fast unstructured CFD code "FaSTAR" 21 , originally developed by JAXA. This solver can handle unstructured grid systems including tetrahedral, triangular prism, square pyramid, and hexahedral meshes and have high flexibility in generating the computational grid. For massive parallel computation on a high-performance computer, the message passing interface (MPI) technique with a domain partition approach is adopted.

The governing equations of the flow field are solved by a cell-centered finite volume approach. The advection fluxes in the Navier-Stakes equations are calculated by the SLAU scheme ${ }^{22}$ with the MUSCL interpolation method for high spatial accuracy. Van leer scheme ${ }^{23}$ is applied as a slope limiter function. On the other hand, the viscous fluxes are basically calculated by averaged value between gradients of neighboring computational cells. Spatial gradient of flow properties is calculated by the weighted Green-Gause method ${ }^{24}$. Time integration is performed by an implicit time-marching method. The governing equation system is transformed into the delta form, and the solution is updated at each time step. The lower-upper symmetric Gauss-Seidel (LU-SGS) ${ }^{25}$ method is employed.

\section{Boundary and Calculation Condition}

Computational domain, boundary condition, and both of grids system for TITANS model with/without AoA are shown in Fig. 3. Unstructured grids can easily genarate computational grids compared with structured grids. Almost all of the mesh in the computational domain is composed of tetrahedral mesh. Thin triangular prism meshes to resolve boundary layer near the wall are partly laminated. The minimum $\mathrm{y}^{+}$at the wall boundary is approximately 0.5 . It is necessary to make fine meshes in front of the vehicle to resolve a shock wave for avoiding numerical instability called "Carbuncle" and abnormal distribution of heat flux on the surface. The grid width in the region of shock wave is $10 \mathrm{~mm}$. Pointwise software is used to generate the grids. Numbers of cells and nodes of computational grids with AoA are approximately $14,800,000$ and 4,200,000, respectively.

Figure 4 shows the reentry trajectory profile of the altitude, reentry velocity, and heat flux versus elapsed time after deorbit for TITANS. Freestream conditions at the inlet are given based on this trajectory profile. The heat flux at the stagnation of the vehicle in this table is calculated by Tauber's model, which is given by

$$
q_{s} \propto \sqrt{\frac{\rho_{\infty}}{R_{N}}} V_{\infty}^{3}
$$

where $q_{s}$ and $R_{N}$ are the heat flux and local nose radius of head capsule. The heat flux gradually increases from approximately $1500 \mathrm{sec}$, and then, reaches a maximum of $204.0 \mathrm{~kW} / \mathrm{m}^{2}$ at an altitude of $88.4 \mathrm{~km}$. The present calculations are performed for 13 cases for altitudes from $94.6 \mathrm{~km}$ to $78.0 \mathrm{~km}$ and for AoA between 0 and 40 degrees, as listed in Table 1. As the outflow conditions at the outlet, all flow properties are determined by the zeroth order extrapolation. No slip for the velocity, fixed temperature of $300 \mathrm{~K}$, and no pressure gradient in the normal direction are assumed on the wall boundary.

American Institute of Aeronautics and Astronautics 
a

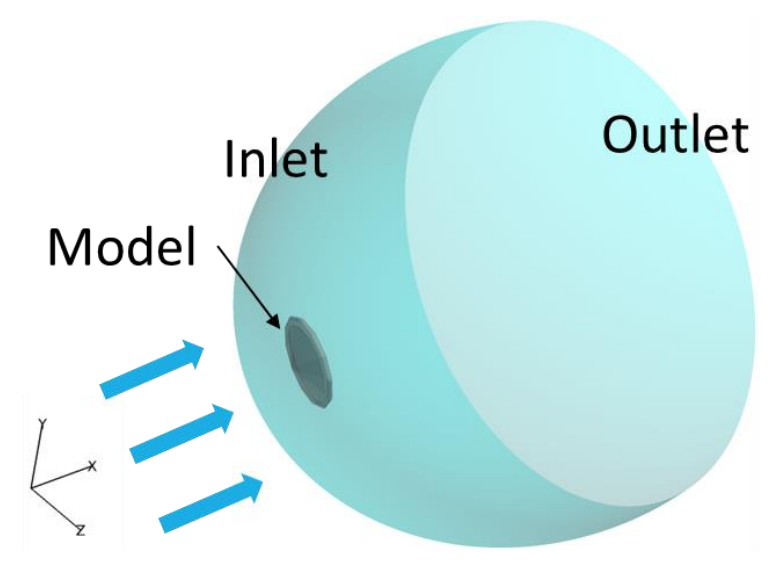

b

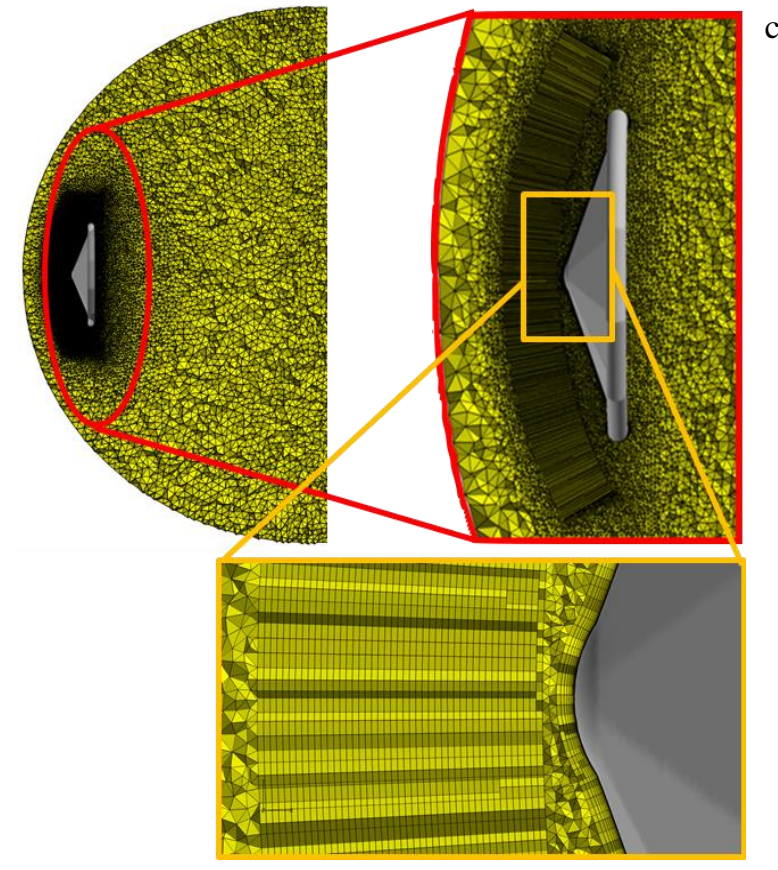

c

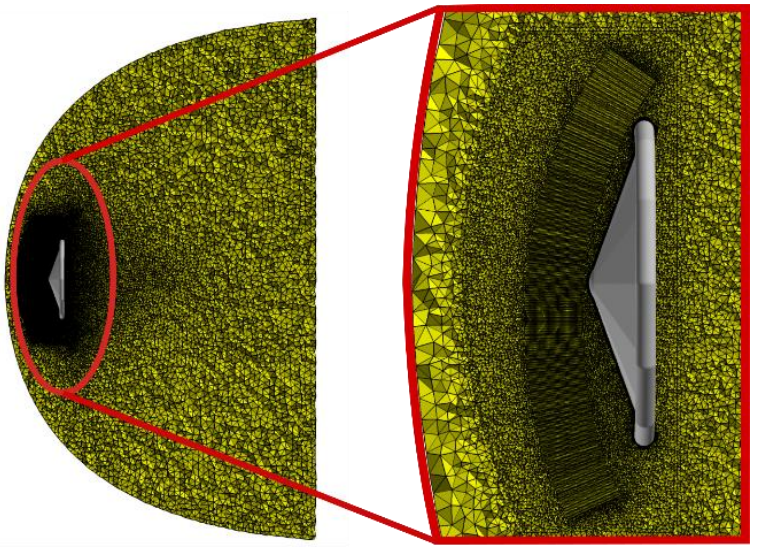

Figure 3. a) Computational domain and boundary conditions, b) Grids system for TITANS without AoA, c) Grids system for TITANS with AoA

Table 1. Freestream conditions

\begin{tabular}{ccccc}
\hline \hline Altitude[km] & 94.6 & 88.4 & 83.2 & 78.0 \\
Maximum heat flux[kW/m²] & 196.9 & 204.0 & 171.5 & 93.14 \\
Mach number & 25.16 & 22.0 & 17.6 & 12.5 \\
Temperature[K] & 190.28 & 187.02 & 192.57 & 202.59 \\
Density[kg/m $\left.{ }^{3}\right]$ & $1.95 \times 10^{-6}$ & $4.86 \times 10^{-6}$ & $1.24 \times 10^{-5}$ & $2.68 \times 10^{-5}$ \\
Velocity[m/s] & 6926.84 & 6037.43 & 4902.92 & 3557.60 \\
AoA [degree] & 0,40 & $0,10,20,30,40$ & 0,40 & 0,40 \\
\hline
\end{tabular}




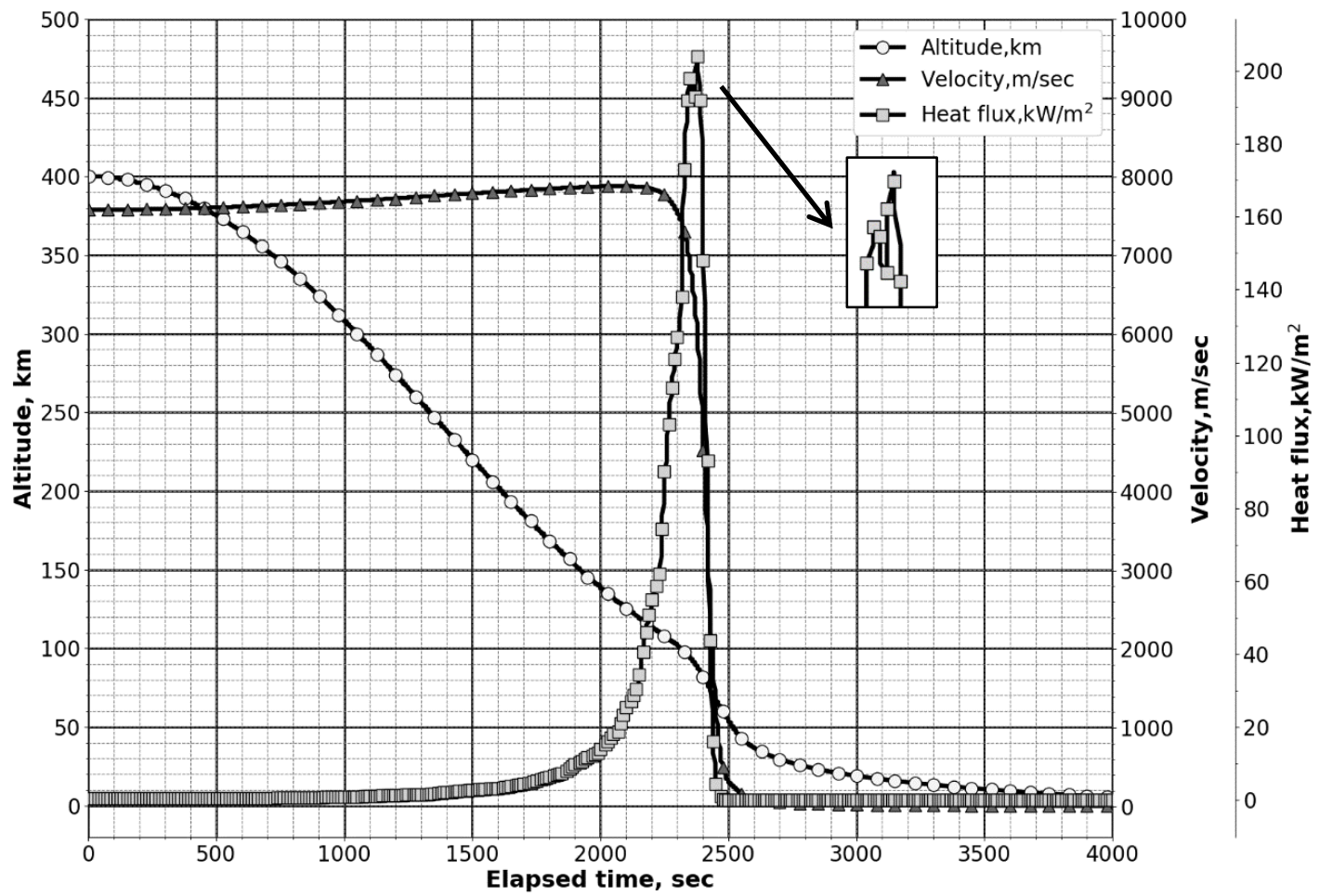

Figure 4. Trajectory profile of TITANS after deorbit

\section{E. Membrane Deformation}

The membrane aeroshell inevitably experiences surface deformation during atmospheric entry, which in turn alters the flowfield around the vehicle and leads to the variations of aerodynamics and aerothermodynamics, due to flexible fabric material. In the current study, membrane deformation is expressed using a particle-based model. It is assumed that the membrane aeroshell is composed of virtual particles and springs connecting the particles. The displacement of particles from their initial state is determined by the aerodynamic force and interactions between neighboring particles as shown in Fig. 5. The virtual particle position $(\boldsymbol{r})$ can be described by

$$
\rho l_{o} S_{o} \frac{d^{2} \mathbf{r}}{d t^{2}}=\mathbf{F}_{E}+\mathbf{F}_{A}
$$

where the elastic force $\mathbf{F}_{E}$ is composed of the tension force $\mathbf{F}_{T}$ and bending force $\mathbf{F}_{B}$ as it is described by

$$
\mathbf{F}_{E}=\mathbf{F}_{T}+\mathbf{F}_{B}
$$

and $\mathbf{F}_{A}$ is the aerodynamic force.

American Institute of Aeronautics and Astronautics 


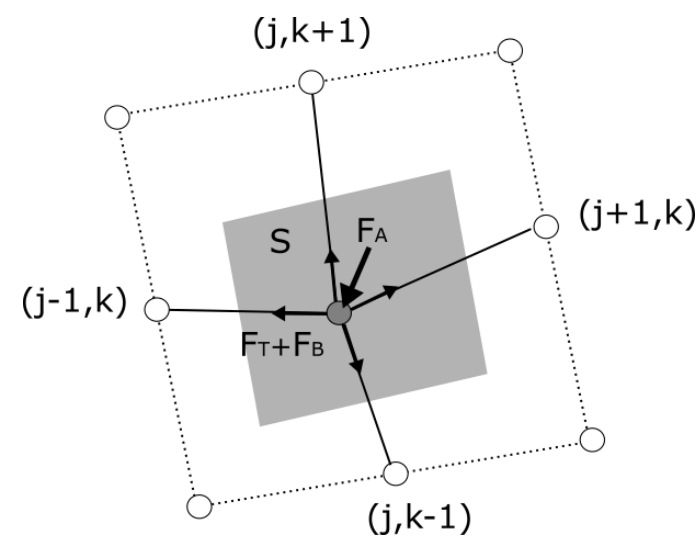

Figure 5. Schematic of the virtual particle network

The $(j+1 / 2, k)$ th component of the tension force $\mathbf{F}_{T}$ is given by

$$
\mathbf{F}_{T(j+1 / 2, k)}=E h_{(j+1 / 2, k)} l_{(j+1 / 2, k)} \frac{\varepsilon_{j(j+1 / 2, k)}+v \varepsilon_{k(j+1 / 2, k)}}{1-v^{2}}
$$

where $E$ and $\boldsymbol{V}$ are Young's modulus and Poisson's ration, respectively. Strains $\varepsilon$ are expressed with length $l_{j}$ and initial length $l_{j 0}$ between virtual particles as

$$
\begin{aligned}
& \varepsilon_{j(j+1 / 2, k)}=\frac{l_{j(j+1 / 2, k)}-l_{j 0(j+1 / 2, k)}}{l_{j 0(j+1 / 2, k)}} \\
& \varepsilon_{k(j+1 / 2, k)}=\frac{l_{k(j+1 / 2, k)}-l_{k 0(j+1 / 2, k)}}{l_{k 0(j+1 / 2, k)}}
\end{aligned}
$$

It is assumed that no compression force works between particles each other in this model, or

$$
\varepsilon_{j(j+1 / 2, k)}=0 \text {, if } l_{j(j+1 / 2, k)}<l_{j 0(j+1 / 2, k)}
$$

The tension force acting on a particle $(j, k)$ is obtained by

$$
\mathbf{F}_{T(j, k)}=\mathbf{F}_{T(j+1 / 2, k)}+\mathbf{F}_{T(j-1 / 2, k)}+\mathbf{F}_{T(j, k+1 / 2)}+\mathbf{F}_{T(j, k-1 / 2)}
$$

The bending force $\mathbf{F}_{B}$ is obtained from the bending moment as it is described by

$$
F_{B(j+, k)}=\frac{M_{j(j, k)}}{l_{j(j+1 / 2, k)}}
$$




$$
F_{B(j-, k)}=\frac{M_{j(j, k)}}{l_{j(j-1 / 2, k)}}
$$

The bending moment is calculated by using

$$
M_{j(j, k)}=E I_{(j, k)} \frac{\Delta \eta_{j(j, k)}+v \Delta \eta_{k(j, k)}}{1-v^{2}}
$$

where $\Delta \eta$ is given by

$$
\Delta \eta_{j(j, k)}=\Delta \eta_{j(j, k)}-\Delta \eta_{j 0(j, k)}
$$

Where $\eta_{j(j, k)}$ is the curvature of neighboring particles $(j+1, k),(j, k),(j-1, k)$, and $\eta_{j 0(j, k)}$ is its initial curvature. The curvature $\eta_{j(j, k)}$ is given by the angle $\alpha_{j, k}$ between position vectors formed by particles $(j+1, k)$ - $(j, k)$ and particles $(j-1, k)-(j, k)$ as described by

$$
\eta_{j(j, k)}=\frac{2 \sqrt{1-\cos ^{2} \alpha_{j(j, k)}}}{\sqrt{l_{j(j+1 / 2, k)}^{2}+l_{j(j-1 / 2, k)}^{2}-2 l_{j(j+1 / 2, k)} l_{j(j-1 / 2, k)} \cos \alpha_{j(j, k)}}}
$$

The area moment of inertia, $I_{(j, k)}$, is calculated by

$$
I_{j(j, k)}=\frac{I_{k(j, k)} h_{(j, k)}^{3}}{12}
$$

The bending force acting on a particle $(j, k)$ is given by

$$
\mathbf{F}_{B(j, k)}=\mathbf{F}_{B(j+, k)}+\mathbf{F}_{B(j-, k)}+\mathbf{F}_{B(j, k+)}+\mathbf{F}_{B(j, k-)}
$$

The bending force acts in the direction normal to the membrane. Thus, a buckling mode can arise in the out-of-plane direction when a strong compression force is exerted in the in-plane direction. The aerodynamic force $\mathbf{F}_{A}$ acting on particle $(j, k)$ is simply given by

$$
\mathbf{F}_{A(j, k)}=p_{(j, k)} S_{(j, k)}
$$

\section{F. Material Properties}

Young's modulus E and Poisson's ration $v$ of Zylon are set to $30 \mathrm{MPa}$ and 0.3 , respectively. These parameters are tuned so that the stretch of the aeroshell because of the membrane deformation corresponds to the flight experimental results, which were constructed based on images taken by onboard JPEG cameras mounted on the SMAAC's backside. Thus, the textile properties of the aeroshell are assumed to be isotropic. The density of the ration of the aerodynamic force to the elastic force of the membrane, is defined by

$$
C_{A E}=\frac{\rho_{\infty} U_{\infty}^{2} L}{E l_{o}}
$$

American Institute of Aeronautics and Astronautics 
where the thickness of the Zylon fabric, $l_{o}$, is $0.155 \mathrm{~mm}$. Thus, the $C_{A E}$ value of the TITANS is on the order of $10^{-2} \sim 10^{-1}$ during atmospheric reentry.

\section{Result and Discussion}

\section{A. Numerical results of flow field and heat flux without $A o A$ at an altitude of $88.4 \mathrm{~km}$}

Distributions of Mach number, translational temperature, and vibrational temperature around TITANS at an altitude of $88.4 \mathrm{~km}$ are shown in Fig. 5. Axial profiles of temperatures and mole fractions along the center line of TITANS from the stagnation point are shown in Fig. 6a and Fig. 6b, respectively. The strong shock wave is formed in front of TITANS. The flow appears to rapidly separate near the top of inflatable torus. This flow structure causes the formation of the large recirculation region behind membrane aeroshell. The separated flow reattaches at a position of approximately $x \sim 2200 \mathrm{~mm}$. No Carbuncle phenomenon that causes numerical instability is observed. Figure $6 \mathrm{a}$ shows that the shock wave formed at the distance of $0.19 \mathrm{~m}$ from the stagnation point causes strong thermal nonequilibrium. The translational temperature increases to $15,300 \mathrm{~K}$ at $0.12 \mathrm{~m}$ and then gradually decreases toward the vehicle surface. In contrast, the vibrational temperature increases to $8,600 \mathrm{~K}$ located at $0.05 \mathrm{~m}$ and then decreases rapidly. The difference of each peak position is related to relaxation time between internal energy modes to reach an equilibrium state. Thermal equilibrium state is observed in the vicinity to the wall. This is because as the number of particle collisions increase and finally energy transfer occurs instantly. Figure $6 \mathrm{~b}$ shows that the dominant chemical reactions are dissociations of molecules because molecular nitrogen and oxygen gradually decreases behind the shock wave, therefore, atomic nitrogen and oxygen are observed near the wall. Molecular oxygen tends to occur dissociations frequently in shock layer compared with nitrogen because dissociation temperature of oxygen is lower than that of molecular nitrogen. Dissociation and ionization reactions rarely occur with decreases in temperature at the wake region of TITANS, although the figure is not shown here.

Figure 7 shows the distribution of heat flux on the front surface of TITANS. Maximum heat flux is $110.3 \mathrm{~kW} / \mathrm{m}^{2}$ on the head capsule because the temperature gradient becomes large due to quite thin shock layer compared with membrane aeroshell and inflatable torus. Maximum heat flux on the stagnation is quite different between the numerical approach and Tauber's model, which shows error of $45.9 \%$ between these methods. This is reason why the Tauber's model depends on the local nose radius of semi-spherical capsule, whereas shape of TITANS is not completely semispherical because of large flare angle of the aeroshell. This feature results in forming an almost normal shock wave.

American Institute of Aeronautics and Astronautics 
a

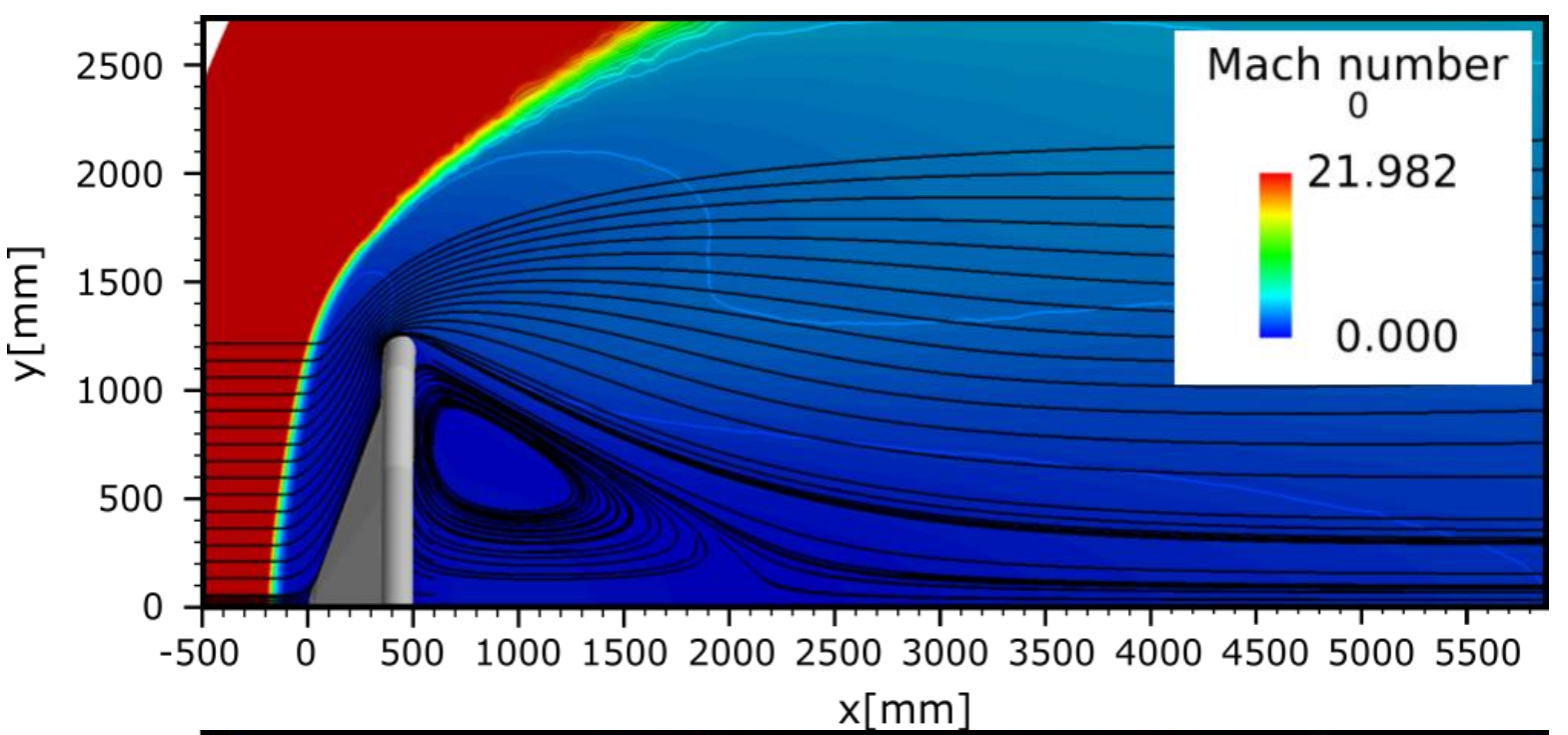

$\mathrm{b}$
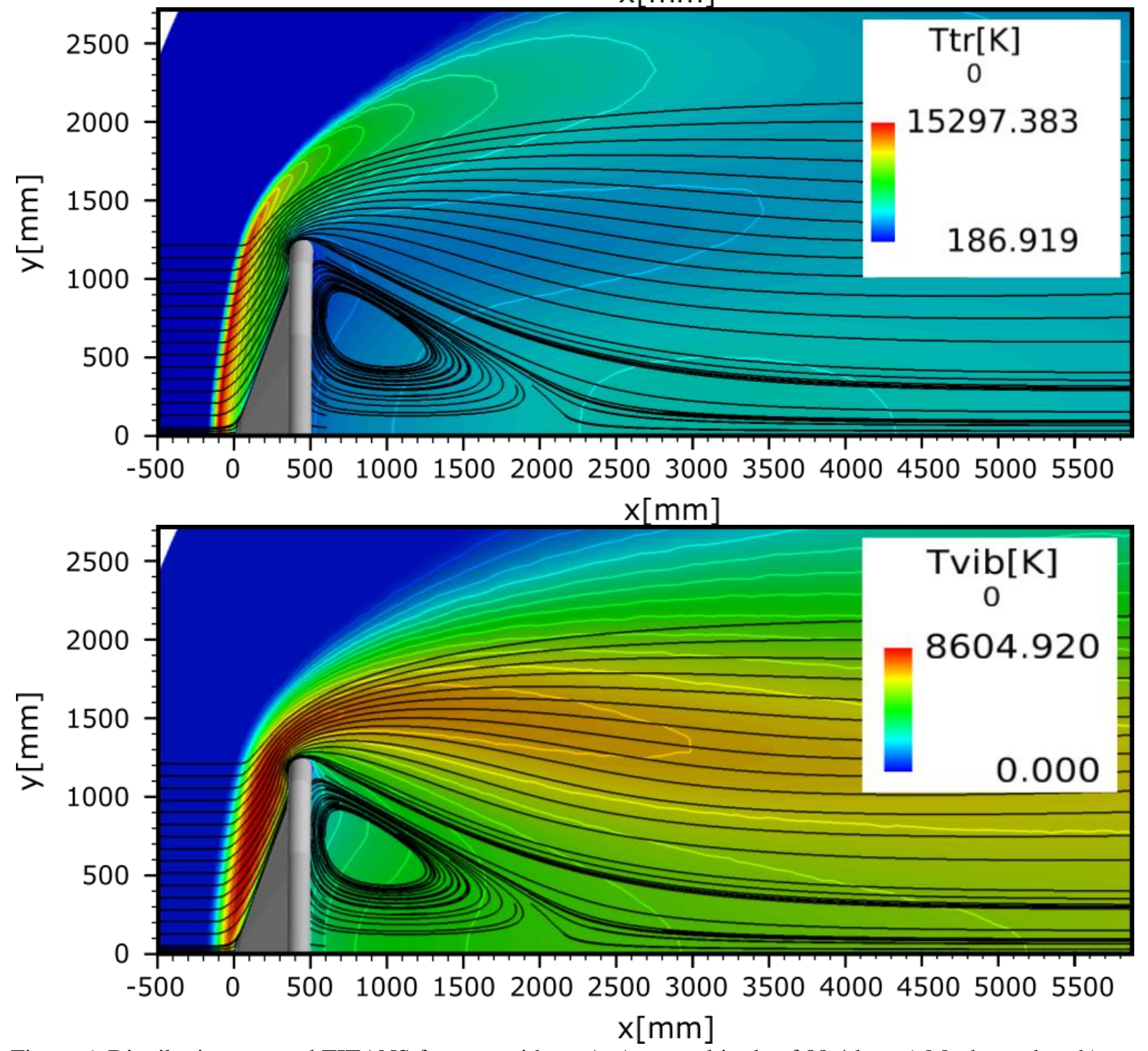

Figure 6. Distributions around TITANS for case without AoA at an altitude of $88.4 \mathrm{~km}$. a) Mach number, b)

Translational temperature, c) Vibrational temperature 
a

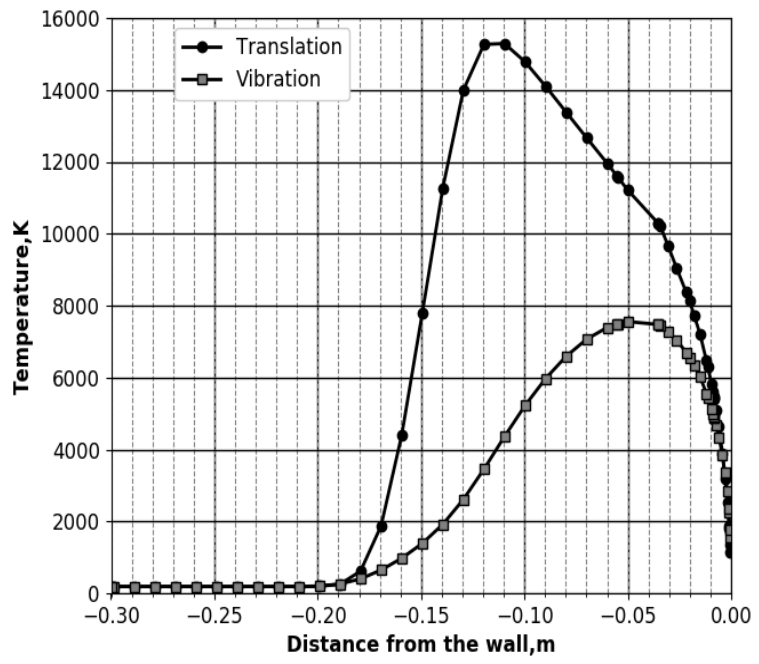

b

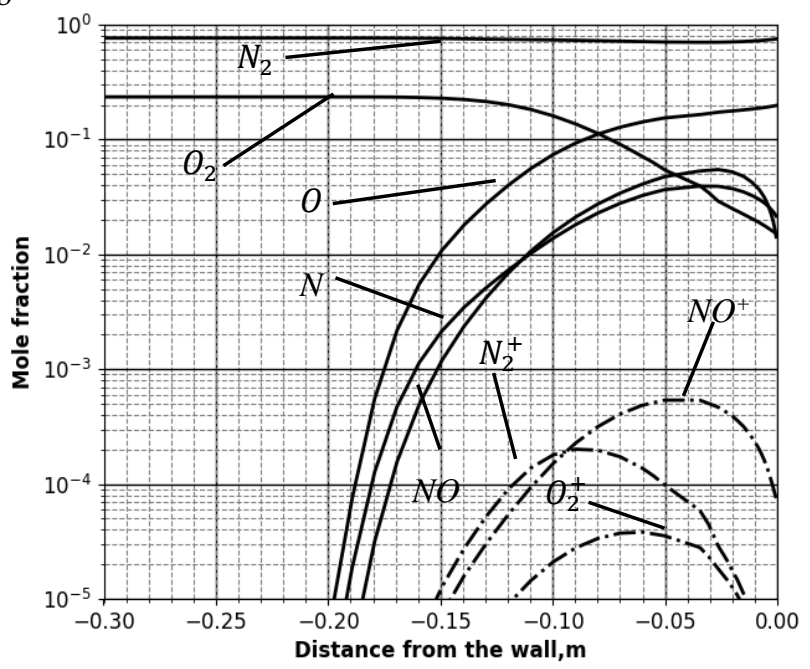

Figure 7. Axial profiles of temperatures and mole fractions along the center axis in front of TITANS for case without AoA at an altitude of $88.4 \mathrm{~km}$

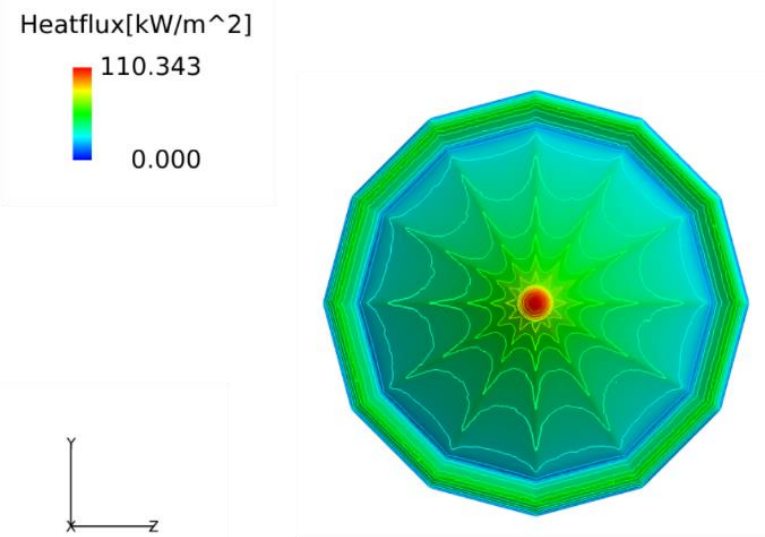

Figure 8. Distribution of heat flux on the surface of TITANS for case without AoA at an altitude of $88.4 \mathrm{~km}$

\section{B. Numerical results of heat flux with AoA}

Figure 9 shows distributions of heat flux for AoAs between 10 and 40. As AoA increases, the heat flux on the upper side of inflatable torus becomes higher than the head capsule. This is because the shock wave approaches to the inflatable torus and temperature gradient dramatically increases. This influence is particularly observed for case of AoA of more than 30 degrees. It is confirmed that the heat flux at the stagnation point for case of AoA of 40 degree is 3.09 times as high as that for 0 degree. Figure 10 shows the profiles of heat flux on the sueface. As confirmed from the heat flux distributions of figure 9, it is also observed in figure 10 that the heat fluxes on the head capsule and the inflatable torus decreases and increases with increase in AoA, respectively. For the case of an AoA of 10 degree, the heat flux distribution is almost the same as the AoA of 0 degree, but the heat flux increment is confirmed as well as the qualitative tendency at other AoA in the inflatable torus on the windward (upper) side. From the distribution for case of AoA of 20 degree, the heat flux tendency differs from that for an AoA of 0 degree. The heat flux of the head capsule and the windward side inflatable torus take almost same values, because distances of the inflatable torus and the capsule from shock wave are almost the same. From the translational temperature distributions at various AoAs shown in Fig. 11, the shock wave for the case of AoA of 20 degree is almost vertically formed against the leewardside inflatable torus. Furthermore, the flow is strongly compressed in this region where the heat flux increases are observed for case of AoA of 20 degree. Although this flow structure can be confirmed even at AoAs of 10 and 30 degrees, there seems to be no influence on an increase in heat flux because this strong compression region exist at other positions in the vicinity of the inflatable torus. The heat flux distribution at an AoA of 40 degree has a region 
where the heat flux value locally increases near $\mathrm{Y}=-1.0 \mathrm{~m}$ of the membrane aeroshell. This is because a high temperature region is formed so as to cover the joint between the inflatable torus part and the membrane aeroshell due to thin shock layer, resulting in a steep temperature gradient. As a result, not only the increase of the maximum heat flux value, but also the local heat flux increment by the change of the flow field structure was also confirmed due to the effect of AoA.

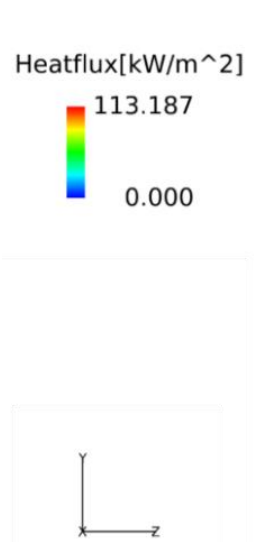

$\mathrm{c}$

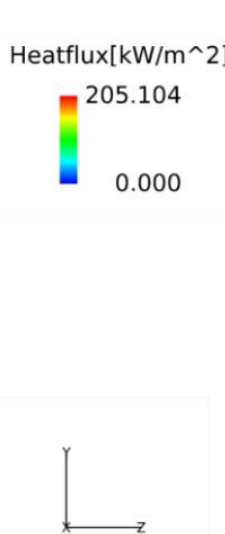

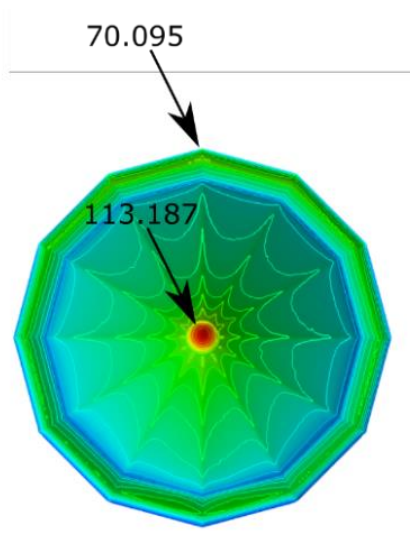

$\mathrm{b}$

d

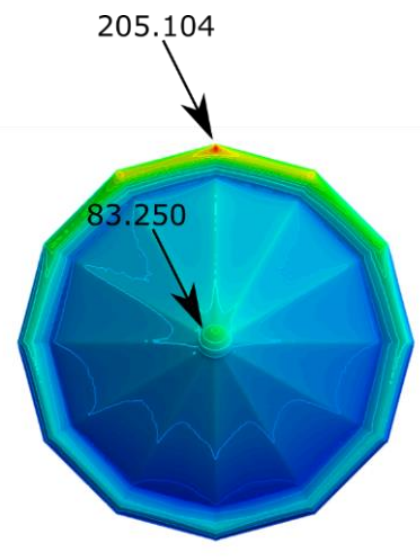

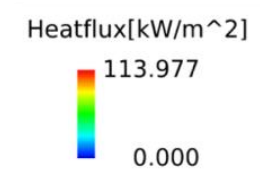

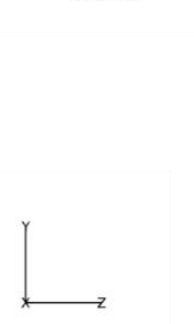

$\mathrm{b}$

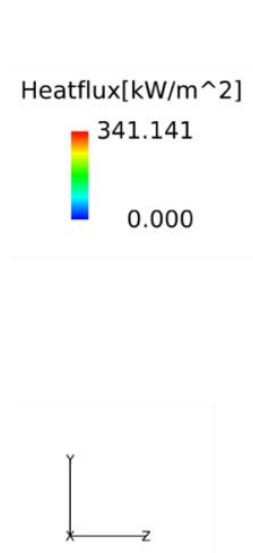

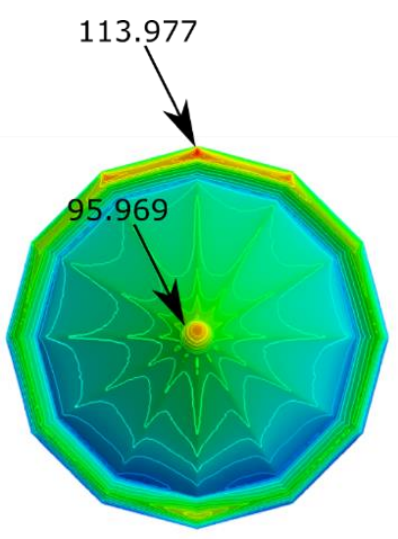

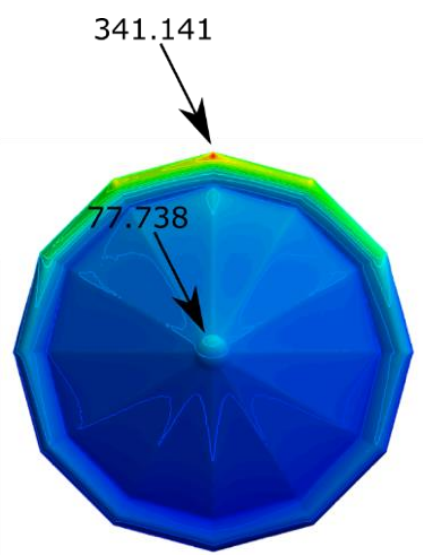

Figure 9. Distribution of heat flux on the surface of TITANS at an altitude of $88.4 \mathrm{~km}$ for cases of a) AoA of 10 degree, b) AoA of 20 degree, c) AoA of 30 degree, d) AoA of 40 degree. 


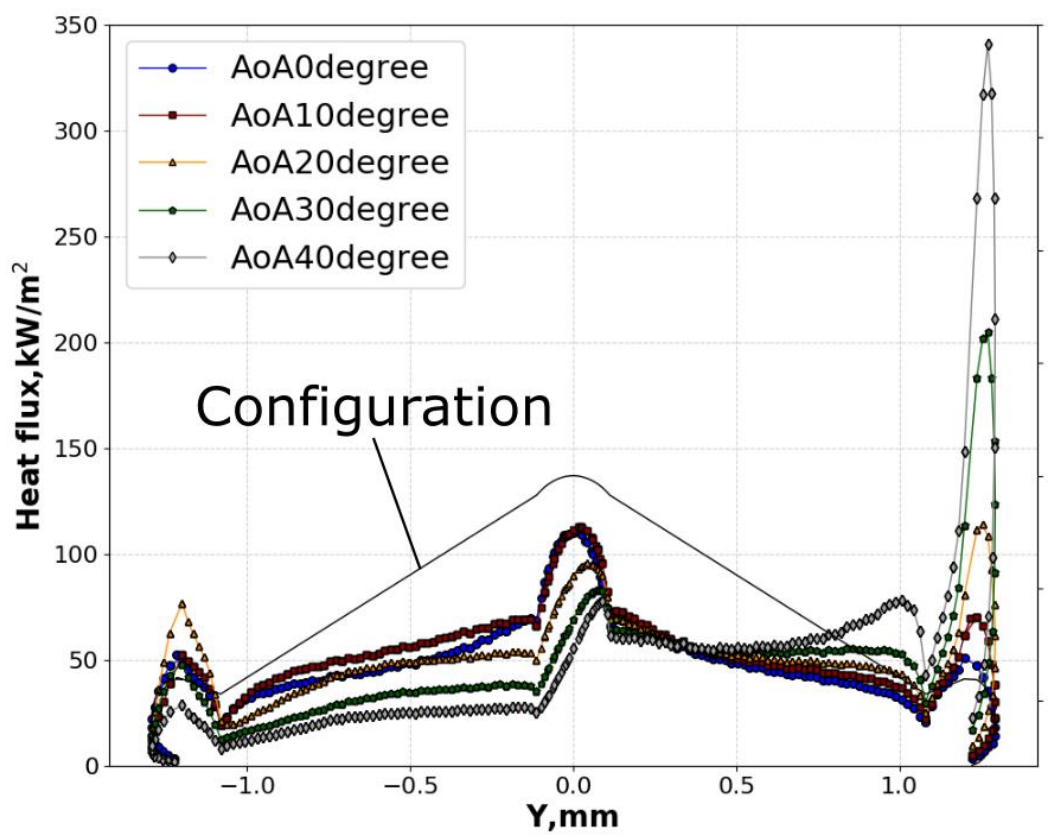

Figure 10. Heat flux profiles at an altitude of $88.4 \mathrm{~km}$ for cases of AoAs between 0 degree and 40 degree

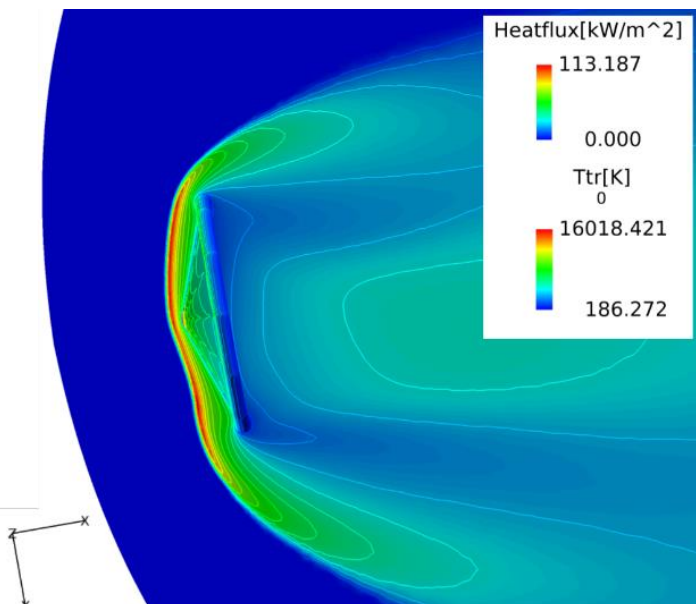

c

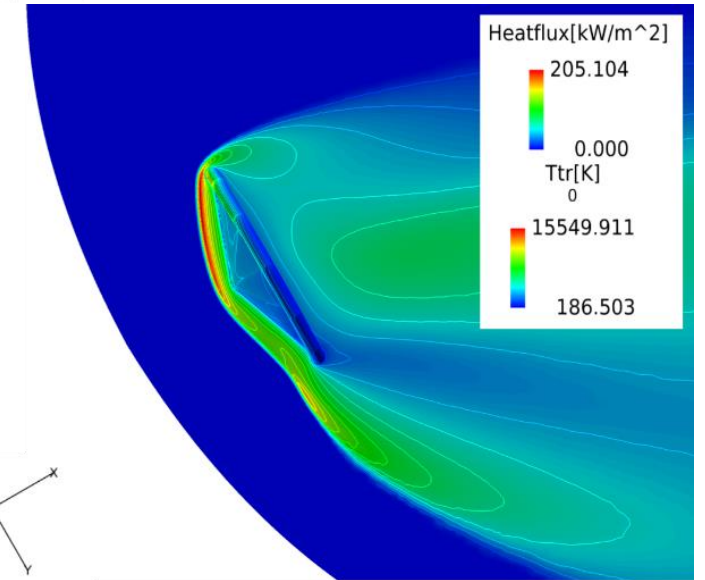

b

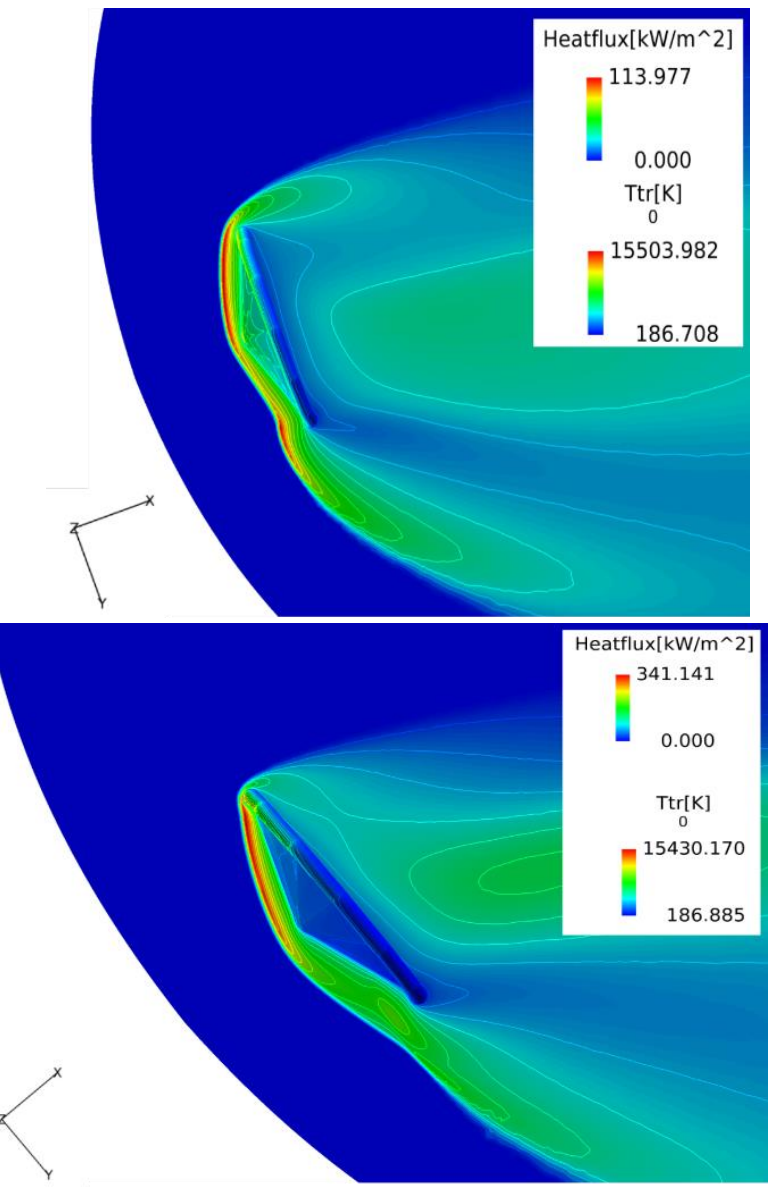

Figure 11. Flow field of Translational temperature around TITANS at an altitude of $88.4 \mathrm{~km}$ for cases of a) AoA of 10 degree, b) AoA of 20 degree, c) AoA of 30 degree, d) AoA of 40 degree.

American Institute of Aeronautics and Astronautics 


\section{Numerical results of heat flux from an altitude of $94.6 \mathrm{~km}$ to $78.0 \mathrm{~km}$}

Figure 12 shows comparison of heat flux histories at stagnation point of TITANS between the Tauber's model and the present numerical simulation. It is clear that the heat flux distribution is different. The numerical simulation results totally estimate the heat flux value lower compared with Tauber's model, and the difference of the maximum change rate is $54 \%$ at an altitude of $83.2 \mathrm{~km}$. This is because the local nose radius in Tauber's model does not correspond to the effective nose radius of TITANS vehicle because of the large flare angle of the membrane aeroshell. As a qualitative trend, the Tauber's model takes the maximum heat flux value at an altitude of $88.4 \mathrm{~km}$, but the heat flux value by numerical simulation reached a maximum at an altitude of $94.6 \mathrm{~km}$. Figure 13 shows heat flux profiles at an altitudes from $94.6 \mathrm{~km}$ to $78.0 \mathrm{~km}$ for cases of AoA bewtween 0 and 40 degrees.

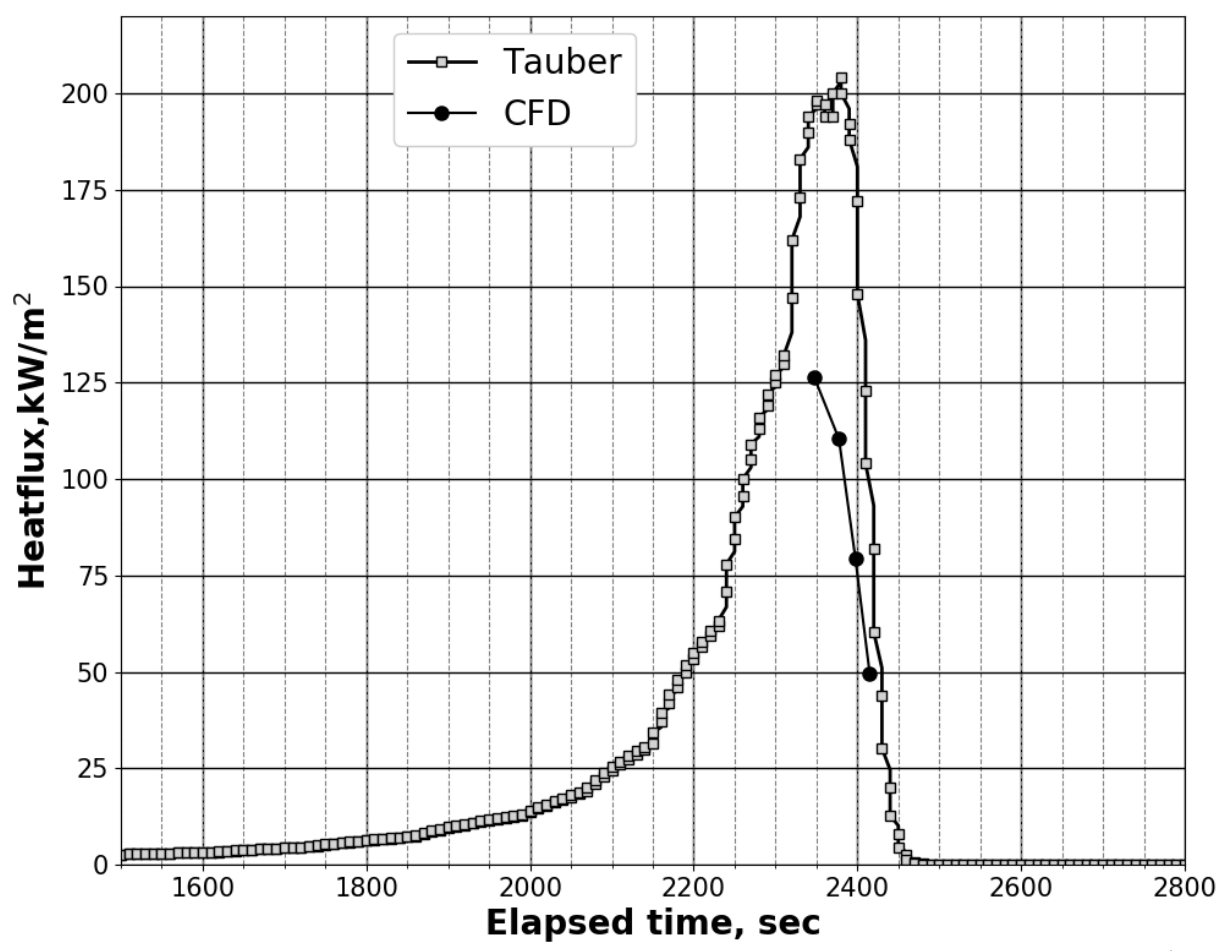

Figure12. Comparison of heat flux histories at the stagnation point of TITANS between the Tauber's model and the present numerical simulation
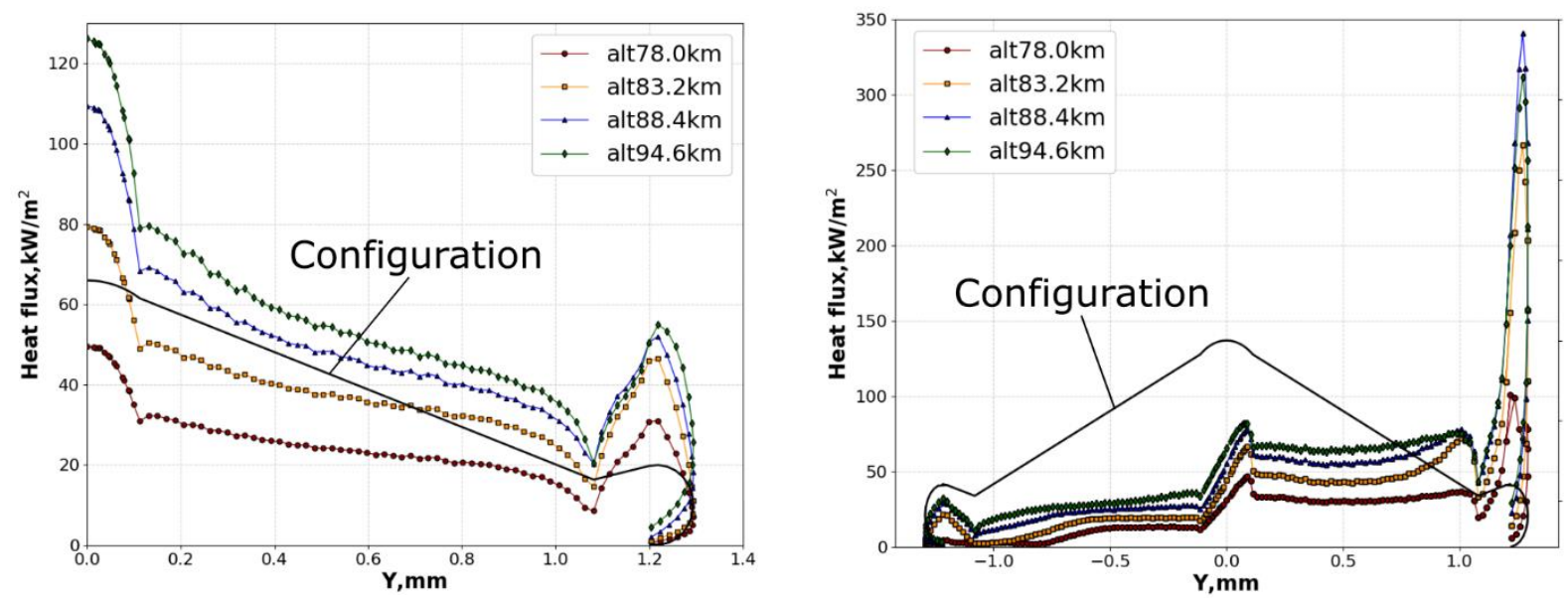

Figure 13. Heat flux profiles at an altitudes from $94.6 \mathrm{~km}$ to $78.0 \mathrm{~km}$ for cases of AoA between 0 and 40 degree

American Institute of Aeronautics and Astronautics 


\section{Numerical results of heat flux by using deformation model}

The effect of membrane deformation on heat flux is investigated at an altitude of $88.4 \mathrm{~km}$ where the heat flux by the Tauber's model reaches peak value in the present trajectory. As a computational result of membrane deformation, the initial shape at the AoA of 0 degree, deformed shape for cases of AoA of 0 and 40 degrees are shown in Figs 14a, $14 \mathrm{~b}$, and $14 \mathrm{c}$, respectively. The pressure distribution obtained by the present numerical simulation when using the initial shape is given as the aerodynamic force to deformation calculation of the membrane aeroshell. Since the aerodynamic force is exerted perpendicularly on the membrane surface, the membrane aeroshell is elongated by pulling in the direction of the outer edge. Because TITANS shape is a dodecagon, forces in the direction of the central axis work for each domain of membrane aeroshell. Therefore, it is expected that wrinkle shapes are formed at the boundary between adjacent surfaces. Actually, it can be confirmed that the wrinkles are formed at the boundary of the surface in the vicinity of the head capsule. In terms of aerodynamic heating, increase in heat flux is concentrated on the wrinkle shapes. The amount of displacement of membrane aeroshell at the AoA of 40 degree has a difference between the windward side (upper side in this figure) and the leeward side (lower side in this figure) because the pressure distribution is not concentric. The membrane aeroshell in windward side is more deformed because of higher pressure distribution. In both deformed models, the membrane aeroshell is displaced such as a catenary curve. The maximum displacements of the membrane aeroshell shape were $6.72 \%$ and $6.64 \%$ relative to the total length of 2.5 $\mathrm{m}$, respectively.

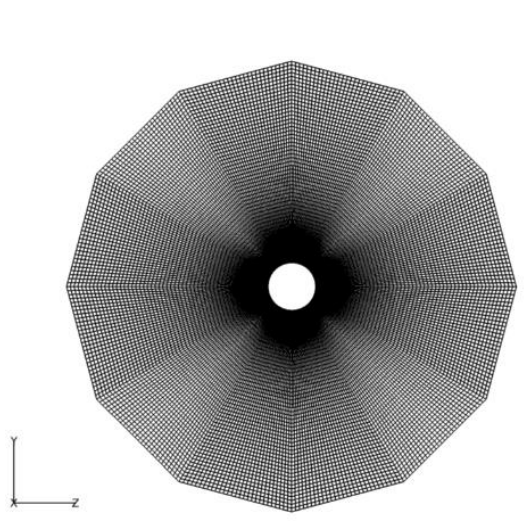

a) Initial shape Displacement[m]

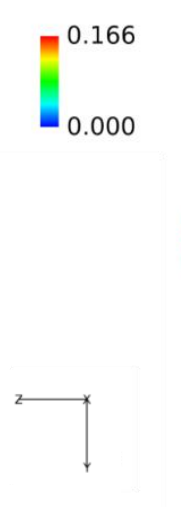

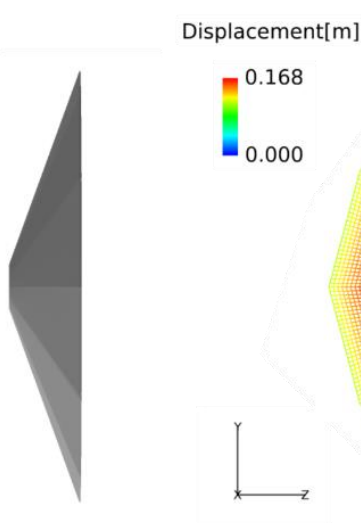

b) Deformed shape for AoA of 0 degree

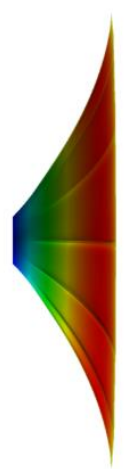

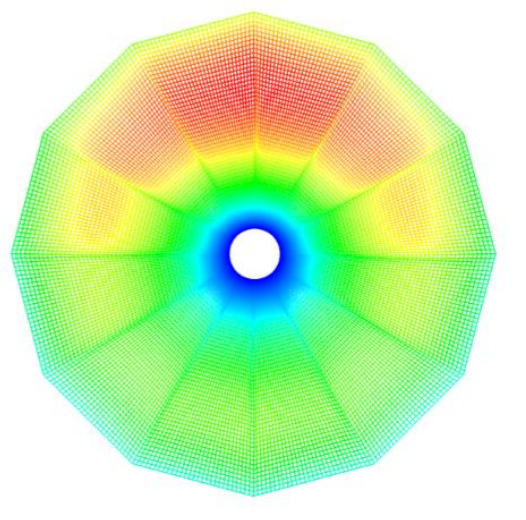

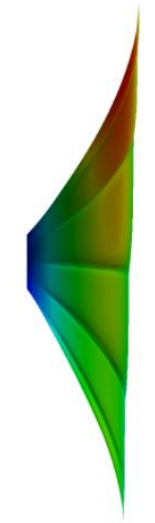

c) Deformed shape for AoA of 40 degree

Figure 14. Configuration of membrane deformation model

Flow field simulation is performed using the membrane deformation model and CFD approach. Figure 15 shows the heat flux distributions for cases of an AoAs of 0 and 40 degree. Figure 16 shows the comparison of the heat flux profiles on the initial shape and deformed shape, focusing on the front surface and wrinkle surface. As expected, the heat flux distribution is different between the initial shape and the deformed shape shown in Figs. 16a, and b. In particular, the difference in the heat flux value on the head capsule part is remarkable as $188.8 \%$. It is suggested that 
the effective nose radius becomes small, and the heat flux on the deformed shape is almost the same as the heat flux calculated by the Tauber's model. In addition, the heat flux decreases in the membrane surface part where the displacement amount is relatively large. Fig. 16b shows that heat flux values at the wrinkle portion are larger than that of the initial shape. Fig. 16c indicates that membrane deformation for case of AoA of 40 degree does not significantly affect the peak heat flux value on the inflatable torus such as case of the AoA of 0 degree. Additionally, increase in heat flux observed at the junction between the membrane aeroshell and the inflatable torus is alleviated because of the large displacement shown in Fig. 16d. The heat flux distribution on the head capsule indicates that the peak position also changes. The heat flux on the wrinkle portion formed on the windward side is higher than that on the initial shape such as the AoA of 0 degree.

Figure 17 shows the Mach number distribution for case of an AoA of 0 degree. In addition, axial profiles of translational and vibrational temperatures along the center axis in front of TITANS are shown in Fig. 18. The shape of the shock wave changes due to influence of membrane deformation. The shock wave formed at upstream region of the head capsule has a similar shape to detachment shock wave seen in front of a blunt body reentry problem. The thickness of the shock layer seems to be thinner. Consequently, this leads to increase in the heat flux on the surface. The recirculation region behind the membrane aeroshell is generated similarly to the initial shape case. However, the reattached position exists more downstream. This is mainly because of difference in separation position on the inflatable torus. As described the flow field structure above mentioned, the shock layer thickness becomes thinner and the thickness is half as long as the shock layer observed by using the initial shape. For this reason, the position where the translational temperature reaches peak value is $-0.11 \mathrm{~m}$ in the initial shape, whereas the peak position for the deformed shape case is $-0.032 \mathrm{~m}$ which shows difference of approximately $70 \%$.

Figure 19 shows distributions of Mach number and translational temperature around deformed-shaped TITANS for case of an AoA of 40 degree at an altitude of $88.4 \mathrm{~km}$. Close-up distributions of translational temperature for both of the shapes are shown in Fig. 20. The shape of shock wave for the case of deformed shape is similar to vertical shock wave because membrane aeroshell largely deforms and relative distance between capsule and inflatable torus in the $\mathrm{x}$-direction becomes closer. Furthermore, high translational temperature region extends near capsule. These changes of flow field result in the difference of heat flux distribution.

a

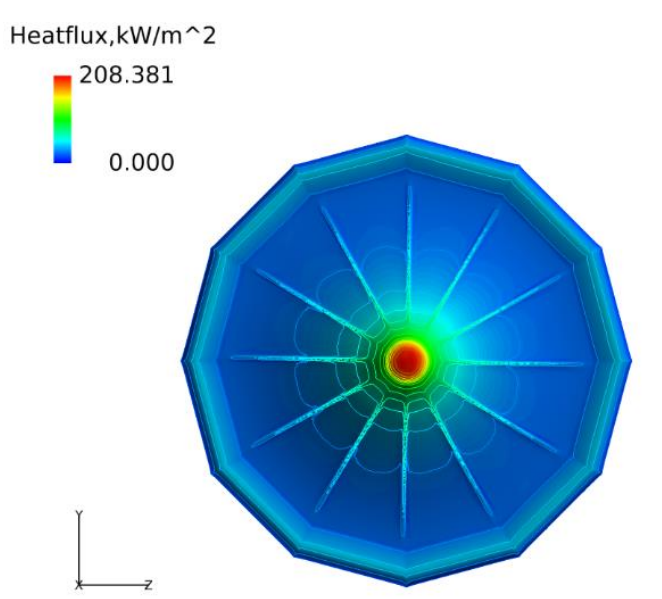

$\mathrm{b}$

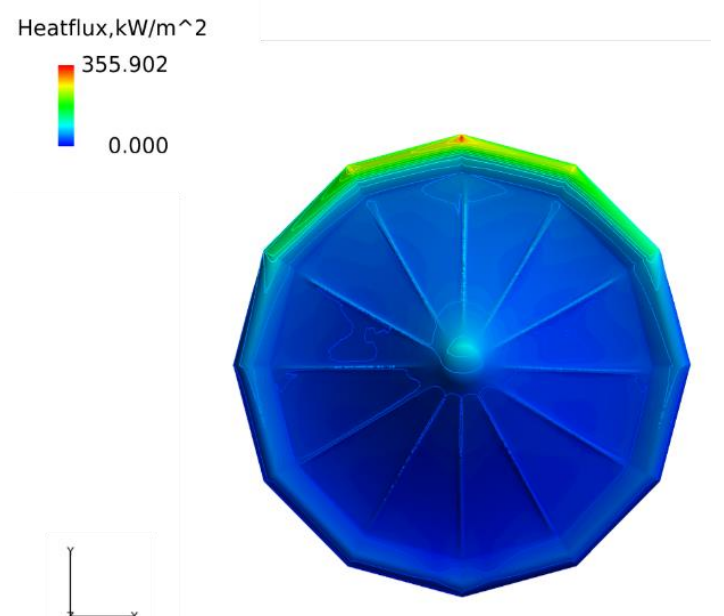

Figure 15. Distributions of heat flux on the surface for the deformed shape at an altitude of $88.4 \mathrm{~km}$ for a) AoA of 0 degree and b) AoA of 40 degree. 


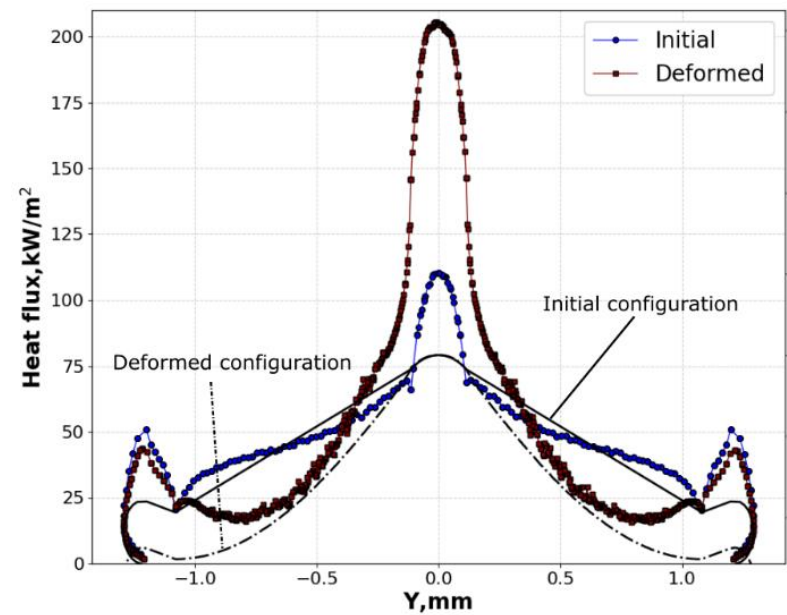

a) Heat flux on the whole surface at AoA of 0 degree

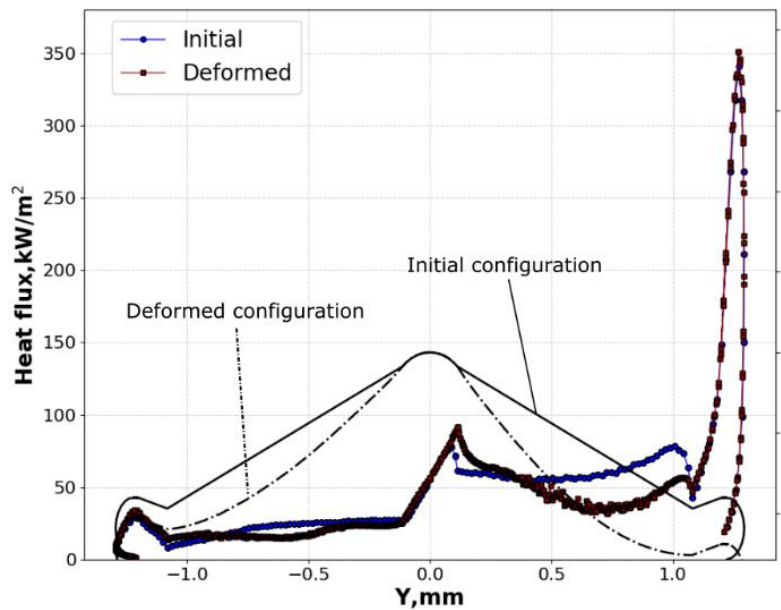

c) Heat flux on the whole surface at AoA of 40 degree

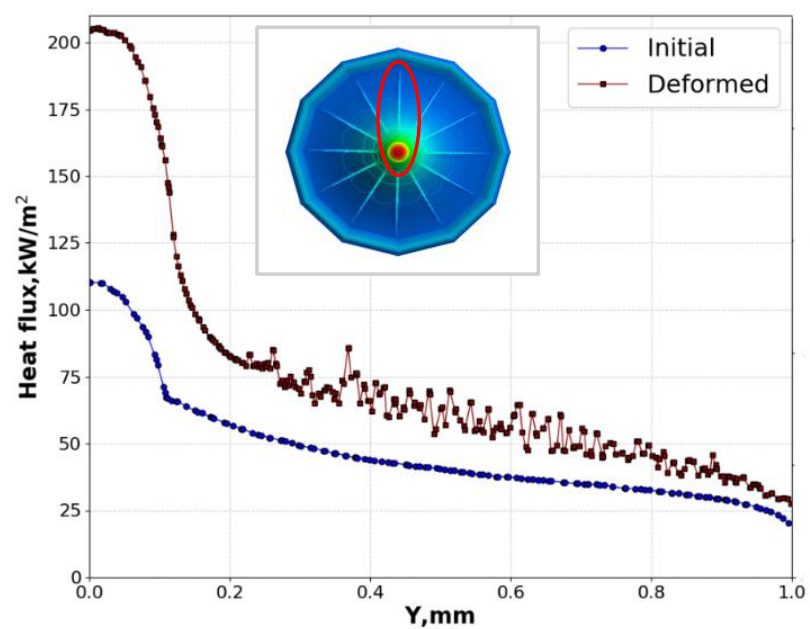

b) Heat flux on a wrinkle at AoA of 0 degree

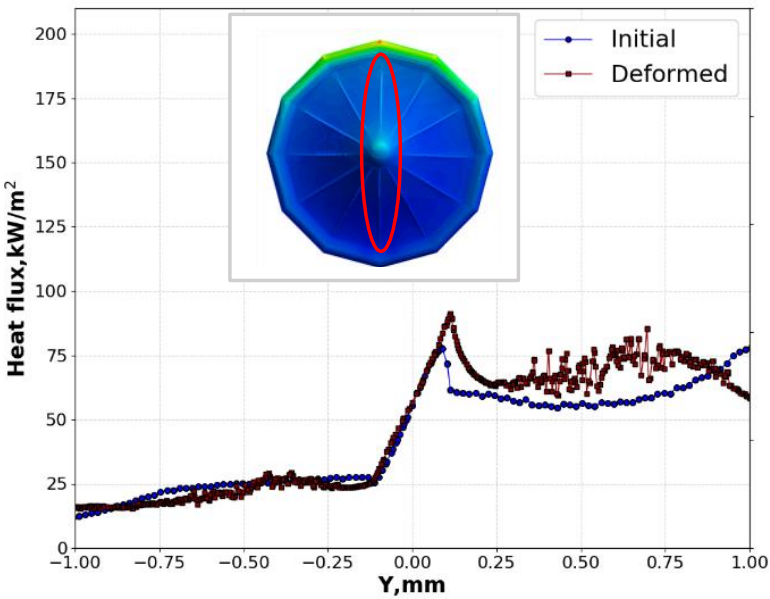

d) Heat flux on a wrinkle at AoA of 40 degree

Figure 16. Profiles of heat flux on the surface of TITANS at an altitude of $88.4 \mathrm{~km}$

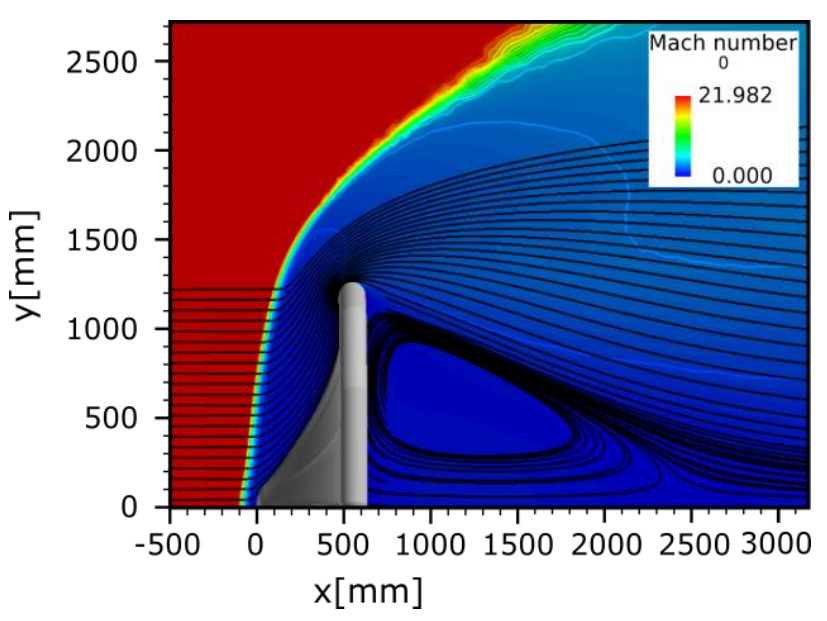

Figure 17. Distribution of Mach number around the deformed shape for case for AoA of 0 degree at an altitude of $88.4 \mathrm{~km}$

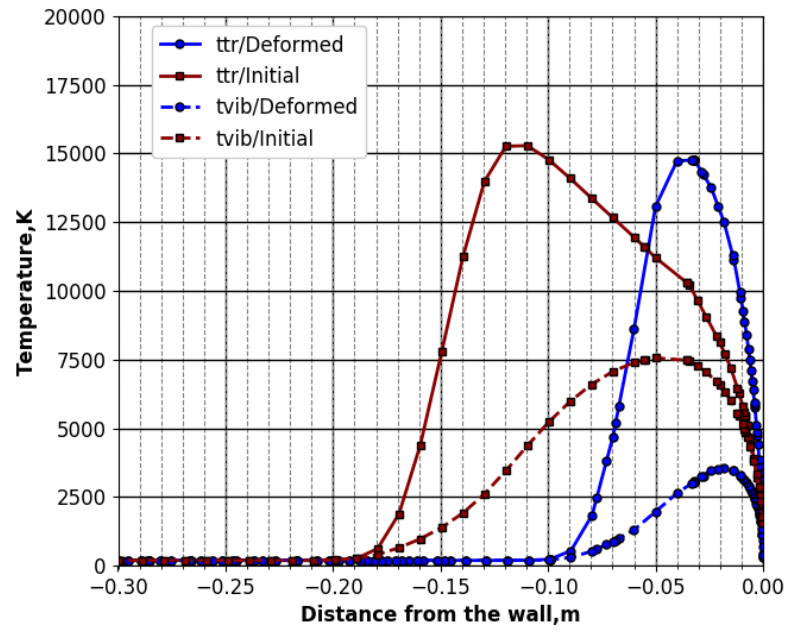

Figure 18. Axial profiles of temperatures along the center axis in front of TITANS for AoA of 0 degree at an altitude of $88.4 \mathrm{~km}$ 

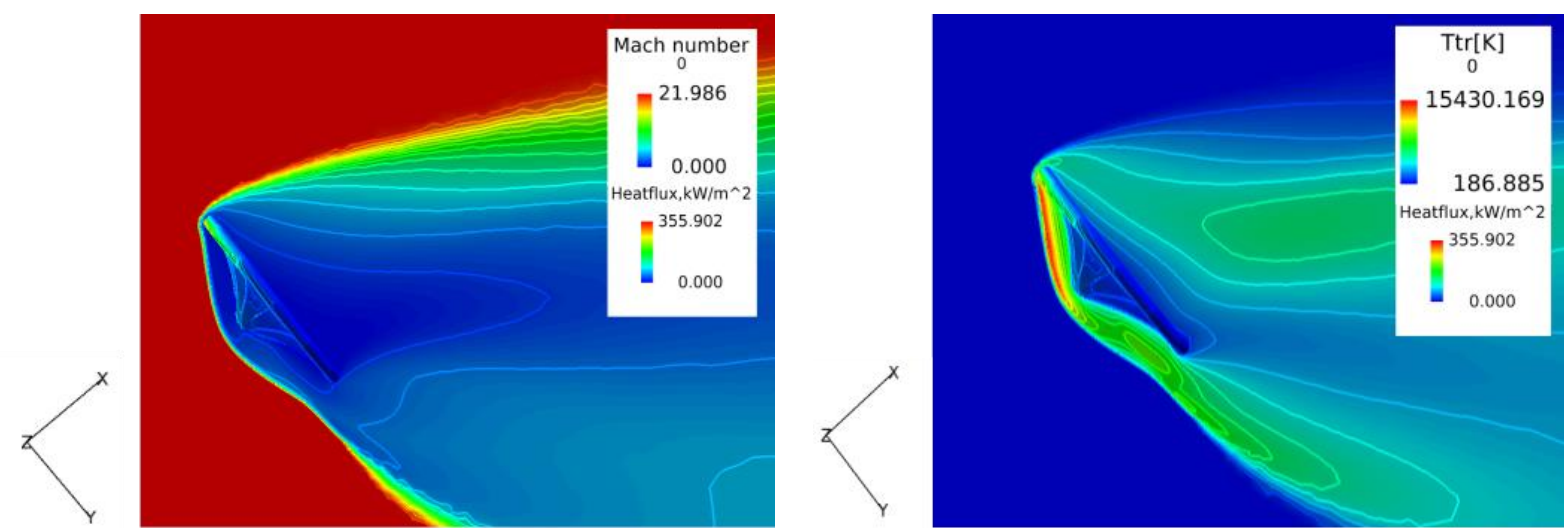

Figure 19. Distributions of Mach number and translational temperature around the deformed shape for case of AoA of 40 degree at an altitude of $88.4 \mathrm{~km}$

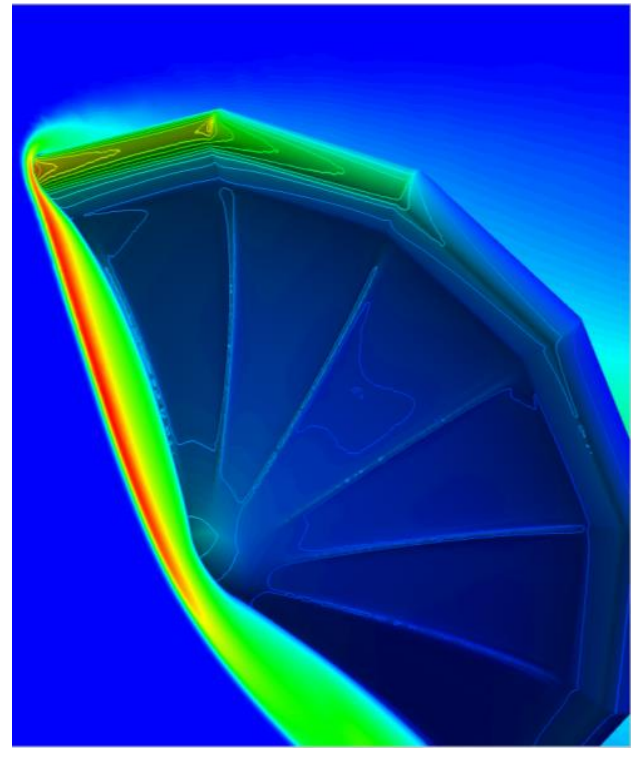

a) Deformed shape

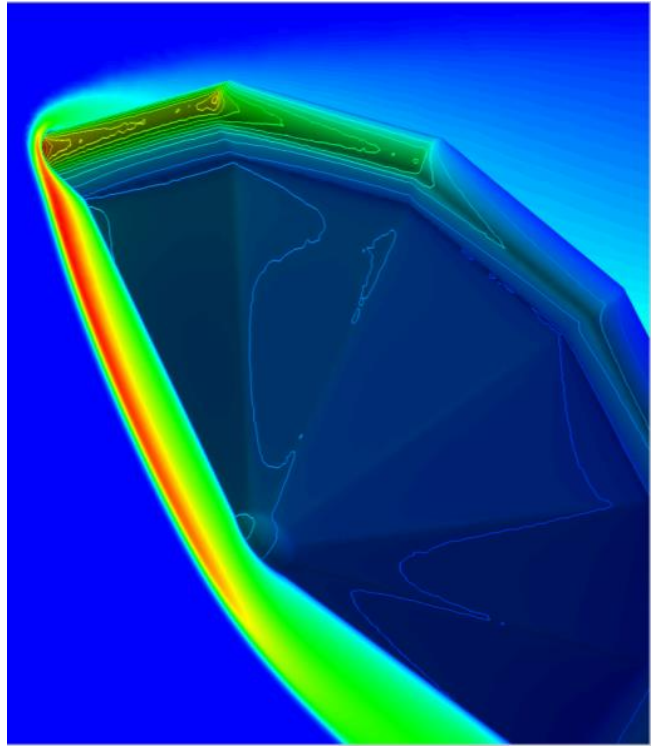

b) Initial shape

Figure 20. Close-up distribution of translational temperature for case of AoA of 40 degree at an altitude of 88.4 $\mathrm{km}$

\section{Conclusion}

We investigated aerodynamic heating for an inflatable reentry vehicle, TITANS, using a flow field simulation approach coupled with structure analysis code. In order to generate computational grids around the TITANS analysis model with reduction of human cost, unstructured grids system was adopted. As applicable solver to this kind of grid system, RG-FaSTAR was used. Main findings were summarized as follow:

A) The basic structure of flow field and distribution of heat flux observed in flare-type membrane inflatable reentry vehicle were reproduced at AoA of 0 degree. Compared with the Tauber's model which is semi-empirical model, maximum heat flux on the stagnation was quite different, whereas there was $45.9 \%$ error due to difference of the local nose radius of semi-spherical capsule.

B) It was indicated that the heat flux at the windward side (the upper side) of inflatable torus increase as AoA increase because the stagnation point changes from the capsule to inflatable torus. It was found that heat flux at the inflatable torus is higher than the capsule for case of more than AoA of 30 degree and heat flux for case of AoA of 40 degree is 3.09 times as high as that for 0 degree. Characteristic heat flux distributions was observed at AoA of 20 and 40 degrees due to changes of flow field structure related to the shape of shock layer. 
C) Heat flux values at the stagnation point of TITANS from altitudes of $94.6 \mathrm{~km}$ to $78.0 \mathrm{~km}$ by present numerical simulation were estimated lower than that by the Tauber's model. The maximum error between these results was $54 \%$ at an altitude of $83.2 \mathrm{~km}$.

D) The maximum displacements of deformed shape of the membrane aeroshell for cases of AoA of 0 and 40 degrees were $6.72 \%$ and $6.64 \%$ relative to the total length of $2.5 \mathrm{~m}$, respectively. Wrinkle shapes were formed at the boundary between adjacent surfaces of each domain. The difference of heat flux value between the deformed and initial shapes on the head capsule part was remarkable as $188.8 \%$ for case of AoA of 0 degree. However, membrane deformation for case of AoA of 40 degree did not significantly affect the peak heat flux value on the inflatable torus such as case of the AoA of 0 degree. It was indicated that the heat flux decreases in the membrane surface part where the displacement amount was relatively large, where increase in heat flux was observed on the wrinkle shapes for both deformed shapes.

\section{Acknowledgments}

The work was supported by JSPS KAKENHI Grant Number 15H04205 and 17K14871. The present computational results were obtained using the fast unstructured CFD code "FaSTAR", which was developed by JAXA. This work was partially supported by "Joint Usage/Research Center for Interdisciplinary Large-scale Information Infrastructures" and "High Performance Computing Infrastructure" in Japan (Project ID: jh160032 and jh170047). The computations were mainly carried out using the computational facilities (HITACHI HA8000-tc/HT210 and FUJITSU PRIMERGY CX400) at the Research Institute for Information Technology, Kyushu University, and the supercomputer system (HITACHI SRI SR16000 model M1) at the Information Initiative Center, Hokkaido University.

\section{References}

${ }^{1}$ K. Yamada, D. Akita, E. Sato, K. Suzuki, T. Narumi, and T. Abe. "Flare-type Membrane Aeroshell Flight Test at Free Drop from a Balloon”. Journal of Spacecraft and Rockets, 46(3):606-614, May-June 2009.

${ }^{2}$ K. Yamada, T. Abe, K. Suzuki, N. Honma, M. Koyama, Y. Nagata, D. Abe, Y. Kimura, A.K. Hayashi, D. Akita, and H. Makino. "Deployment and Flight Test of Inflatable Membrane Aeroshell using Large Scienti_c Balloon". AIAA Paper 20112579, 2011.

${ }^{3}$ K. Yamada, Y. Kato, and T. Abe. "Numerical Simulation of Hypersonic Flow around Flare-Type Aeroshell with Inflatable torus Frame". In Proceedings of 6th Asia Workshop on Computational Fluid Dynamics, Kashiwa, Japan, March 162009.

${ }^{4}$ K. Yamada, T. Sonoda, K. Nakashino, and T. Abe. "Structural Strength of Flare-type Membrane Aeroshell Supported by Inflatable Tours against Aerodynamic Force". In Proceedings of 28th International Symposium on Space Technology and Science, ISTS 2011-c-34, Okinawa, Japan, June 5 - 122011.

${ }^{5}$ Y. Takahashi, K. Yamada, and T. Abe. "Radio Frequency Blackout Possibility for an Inflatable Reentry Vehicle". AIAA Paper 2012-3110, 2012.

${ }^{6}$ Y. Takahashi, K. Yamada, and T. Abe. "Examination of Radio Frequency Blackout for an Inflatable Vehicle during Atmospheric Reentry". Journal of Spacecraft and Rockets, 51(2):430-441, March 2014.

${ }^{7}$ K. Yamada, T. Abe, K. Suzuki, O. Imamura, and D. Akita. "Reentry Demonstration Plan of Flare-type Membrane Aeroshell for Atmospheric Entry Vehicle using a Sounding Rocket". AIAA Paper 2011-2521, 2011.

${ }^{8}$ K. Yamada, Y. Nagata, N. Honma, D. Akita, O. Imamura, T. Abe, and K. Suzuki. "Reentry Demonstration Deployable and Flexible Aeroshell for Atmospheric-Entry Vehicle using Sounding Rocket". In Proceedings of 63th International Astronau-tical Congress, AC-12-D2.3.3, Naples, Italy, October 1 - 52012.

${ }^{9}$ K. Yamada, Y. Nagata, T. Abe, K. Suzuki, O. Imamura, and D. Akita. "Reentry Demonstration of Flare-type Membrane Aeroshell for Atmospheric Entry Vehicle using a Sounding Rocket”. AIAA Paper 2013-1388, 2013.

${ }^{10}$ Y. Nagata, K. Yamada, T. Abe, and K Suzuki, “Attitude Dynamics for Flare-type Membrane Aeroshell Capsule in Reentry Flight Experiment”. AIAA Paper 2013-1285, 2013.

${ }^{11}$ Y. Takahashi, K. Yamada, T. Abe, and K. Suzuki, “Aerodynamic Heating around Flare-type Membrane Inflatable Vehicle in Suborbital Reentry Demonstration Flight”. Journal of Spacecraft and Rockets, 52(6):1530-1541, Novermber 2015.

${ }^{12}$ K. Yamada, T. Moriyoshi, K. Matsumaru, H. Kanemaru, T. Araya, K. Suzuki, O. Imamura, D. Akita, Y. Nagata, Y. Shoji, Y. Takahashi, Y. Watanabe, T. Abe, "Re-entry Nano-Satellite with Gossamer Aeroshell and GPS/Iridium deployed from ISS", Joint Conference: $31^{\text {st }}$ ISTS (International Symposium on Space Technology and Science), $26^{\text {th }}$ ISSFD (International Symposium on Space Flight Dynamics), and $8^{\text {th }}$ NSAT (Nano-Satellite Symposium), 2017-f-021, Matsuyama, Ehime, June 3-9, 2017.

${ }^{13}$ M. Matsunaga, Y. Takahashi, N. Oshima, K. Yamada, "Aerodynamic Heating Prediction of an Inflatable Reentry Vehicle in a Hypersonic Wind Tunnel”. AIAA Scitech Forum, AIAA Paper-2017-0263, 2017.

American Institute of Aeronautics and Astronautics 
${ }^{14}$ Y.Takahashi, K. Yamada, T. Abe, K. Suzuki, “Aerodynamic Heating around Flare-type Membrane Inflatable Vehicle in Suborbital Reentry Demonstration Flight"

${ }^{15}$ R.N. Gupta, J.M. Yos, R.A. Thompson, and K.P. Lee. "A Review of Reaction Rates and Thermodynamic and Transport Properties for an 11-Species Air Model for Chemical and Thermal Nonequilibrium Calculations to 30000 K". NASA RP-1232, Aug. 1990.

${ }^{16}$ M. Fertig, A. Dohr, and H.H. Fru hauf. "Transport Coefficients for High-Temperature Nonequilibrium Air Flows". Journal of Thermophysics and Heat Transfer, 15(2):148- 156, April-June 2001.

${ }^{17}$ C. Park. "Assessment of a Two-Temperature Kinetic Model for Dissociating and Weakly Ionizing Nitrogen". Journal of Thermophysics and Heat Transfer, 2(1):8-16, Jan.-March 1988.

${ }^{18}$ C. Park. Nonequilibrium Hypersonic Aerothermodynamics. Wiley, New York, 1990.

${ }^{19}$ R.N. Gupta, J.M. Yos, R.A. Thompson, and K.P. Lee. "A Review of Reaction Rates and Thermodynamic and Transport Properties for an 11-Species Air Model for Chemical and Thermal Nonequilibrium Calculations to 30000 K". NASA RP-1232, Aug. 1990.

${ }^{20}$ Y. Takahashi. "Advanced validation of CFD-FDTD combined method using highly applicable solver for reentry blackout Prediction". Journal of Physics D: Applied Physics, 49(1):015201, 2016.

${ }^{21}$ A. Hashimoto, K. Murakami, T. Aoyama, K. Ishiko, M. Hishida, M. Sakashita, and P.R. Lahur. "Toward the Fastest Unstructured CFD". AIAA Paper 2012-1075, 2012.

${ }^{22}$ E. Shima and K. Kitamura, "Parameter-Free Simple Low-Dissipation AUSM-Family Scheme for All Speeds". AIAA Journal, Vol. 49, No. 8, 2011, pp. 1693-1709.

${ }^{23}$ M. Hishida, A. Hashimoto, K. Murakami, T. Aoyama. "A new slope limiter for fast unstructured CFD solver FaSTAR", JAXA-SP-10-012, 2010

${ }^{24}$ D. J. Mavriplis, "Revisiting the Least-Squares Procedure for Gradient Reconstruction on Unstructured", AIAA Paper, 20033986, 2003.

${ }^{25}$ Jameson, A., Yoon, S., "Lower-Upper Implicit Schemes with Multiple Grids for the Euler Equations", AIAA Journal, Vol. 25, No. 7, 929-935, 1987

American Institute of Aeronautics and Astronautics 